TRANSACTIONS OF THE

AMERICAN MATHEMATICAL SOCIETY

Volume 357, Number 8, Pages 2975-3011

S 0002-9947(05)03799-2

Article electronically published on March 25, 2005

\title{
REGULAR DOMAINS IN HOMOGENEOUS GROUPS
}

\author{
ROBERTO MONTI AND DANIELE MORBIDELLI
}

\begin{abstract}
We study John, uniform and non-tangentially accessible domains in homogeneous groups of steps 2 and 3 . We show that $C^{1,1}$ domains in groups of step 2 are non-tangentially accessible and we give an explicit condition which ensures the John property in groups of step 3.
\end{abstract}

\section{INTRODUCTION}

This paper deals with the study of John, uniform and non-tangentially accessible domains in homogeneous groups (Carnot groups) of steps 2 and 3 endowed with the Carnot-Carathéodory distance induced by a system of generators of their Lie algebra. Such regular domains are strongly related to the global embedding properties of Sobolev spaces and to the study of the boundary behavior of harmonic functions with respect to sub-elliptic Laplacians.

John domains have been introduced by John Joh. It is known from the classical theory of Sobolev spaces that if $\Omega \subset \mathbb{R}^{n}$ is a John domain and $\nabla u \in L^{p}(\Omega)$, then $u \in L^{p n /(n-p)}(\Omega)$ (see the references $[\mathrm{Be}$ and $[\mathrm{Bo}$ ). In the setting of homogeneous groups it is known that if $\Omega$ is a John domain with respect to the Carnot-Carathéodory distance, the Sobolev-Poincaré inequality

$$
\left(\int_{\Omega}\left|u-u_{\Omega}\right|^{p^{*}} d x\right)^{1 / p^{*}} \leq C\left(\int_{\Omega}|X u|^{p} d x\right)^{1 / p}
$$

holds for all $u \in C^{1}\left(\mathbb{R}^{n}\right)$, where $u_{\Omega}$ is the average of $u$ over $\Omega, X u=\left(X_{1} u, \ldots, X_{m} u\right)$ is the sub-elliptic gradient of $u$ with respect to the vector fields $X_{1}, \ldots, X_{m}$ generating the Lie algebra of the group, the integer $Q$ is the homogeneous dimension of the group, $1 \leq p<Q$ and $p^{*}=p Q /(Q-p)$. Different forms of inequality (1.1) have been proved in [FLW], GN1 and [HK] and they are a consequence of the Poincaré inequality proved by Jerison in [J] and of a chaining argument (relations between John condition and chaining properties are studied in [BKL], GN1] and [HK]).

Uniform domains (also known as $(\epsilon, \delta)$ domains) are a sub-class of John domains. The definition of uniform domain is due to Martio and Sarvas [MS] and to Jones Jon. In the latter paper an extension theorem for Sobolev functions in uniform domains is proved, and the theorem is generalized in [VG] and [GN2] to the setting of Carnot-Carathéodory spaces.

Received by the editors February 1, 2002.

2000 Mathematics Subject Classification. Primary 43A80.

Key words and phrases. John domains, non-tangentially accessible domains, homogeneous groups.

The first author was supported by Trento University, Italy, and Bern University, Switzerland.

(C)2005 American Mathematical Society Reverts to public domain 28 years from publication 
A sub-class of uniform domains are non-tangentially accessible domains (briefly NTA domains) which, in the Euclidean case, were introduced by Jerison and Kenig [JK] in connection with the study of the boundary behavior of harmonic functions. The notion of NTA domain can be formulated in terms of the Carnot-Carathéodory metric associated with a sub-elliptic Laplacian $\mathcal{L}=\sum X_{j}^{2}$ and used to prove a Fatou type theorem for non-negative weak solutions $u$ of the equation $\mathcal{L} u=0$ (see [CG] and see also $[\mathrm{W}]$ ). In connection with harmonic measures for sub-elliptic Laplacians, a class of "cuspidal Harnack domains" has been recently studied in [FF1] and [FF2].

It is known that inequality (1.1) may fail even for smooth domains in CarnotCarathéodory spaces. See [J] Section 6], where a counterexample is given. The natural problem that arises is to find explicit examples of domains where inequality (1.1) holds. This problem becomes very intriguing for homogeneous groups of step larger than 2 (in the step-two case more refined properties will be established; see the discussion below). In this paper we give a first answer to this problem.

In groups of step 3 we have found a differential condition on the boundary near characteristic points that seems to control in a sharp way the flatness behavior of the surface in order to have regularity. Our result gives a sufficient condition for the John condition and thus for (1.1). Precisely, consider two vector fields $X_{1}$ and $X_{2}$ in $\mathbb{R}^{4}$ generating the Lie algebra of a homogeneous group of step 3 with non-trivial commutators $\left[X_{1}, X_{2}\right]=X_{3}$ and $\left[X_{1}, X_{3}\right]=\left[X_{2}, X_{3}\right]=X_{4}$. Here $X_{j}=\partial / \partial x_{j}$, $j=1, \ldots, 4$, at the origin (a complete description of this structure is given in Section 4). In this setting we prove the following result:

Theorem 1.1. If $\Omega \subset \mathbb{R}^{4}$ is an admissible domain, then it is a John domain (with respect to the Carnot-Carathéodory distance generated by $X_{1}$ and $X_{2}$ ).

The definition of admissible domain will be given in a detailed way in Section 4 . We observe here that the key property that an admissible domain $\Omega$ should enjoy is the following "flatness" condition. If $\Omega=\left\{x \in \mathbb{R}^{4}: \Phi\left(x_{1}, x_{2}, x_{3}, x_{4}\right)>0\right\}$, we require that for all points in $\partial \Omega$ the estimate

$$
\left|X_{1}^{2} \Phi\right|+\left|X_{2}^{2} \Phi\right|+\left|\left(X_{1} X_{2}+X_{2} X_{1}\right) \Phi\right| \leq k\left(\left|X_{1} \Phi\right|^{1 / 2}+\left|X_{2} \Phi\right|^{1 / 2}+\left|X_{3} \Phi\right|\right)
$$

holds for a suitable positive constant $k$ (see Lemma 4.3 and Theorem 4.4). This condition implies that at characteristic points of second type, that is, characteristic points where also $X_{3} \Phi$ vanishes (see Section 4 ), the second derivatives of $\Phi$ along $X_{1}$ and $X_{2}$ must also vanish. In particular, if (1.2) holds and $\Omega$ agrees near the origin with the set $\left\{x \in \mathbb{R}^{4}: x_{4}>\varphi\left(x_{1}, x_{2}, x_{3}\right)\right\}, \varphi(0)=0, \nabla \varphi(0)=0$, then (see Lemma 4.3) it should be $\left|\varphi\left(x_{1}, x_{2}, x_{3}\right)\right| \leq k\left(\left|x_{1}\right|+\left|x_{2}\right|+\left|x_{3}\right|^{1 / 2}\right)^{3}$. This flatness condition is not ensured by Euclidean regularity. In Example 5.2 we also explicitly construct admissible domains.

On the other hand, we have the following necessary condition, whose proof is contained in Proposition 5.6.

Theorem 1.2. If a domain $\Omega$ locally agrees near the origin with the set $\left\{x \in \mathbb{R}^{4}\right.$ : $\left.x_{4}>\varphi\left(x_{1}, x_{2}, x_{3}\right)\right\}$ and $\left|\varphi\left(x_{1}, x_{2}, x_{3}\right)\right| \leq k\left(\left|x_{1}\right|+\left|x_{2}\right|+\left|x_{3}\right|^{1 / 2}\right)^{\gamma}$ for some $\gamma$ strictly larger than 3, then the Sobolev-Poincaré inequality (1.1) does not hold in $\Omega$.

This shows two surprising facts: (i) if a domain $\Omega$ locally agrees near the origin with the half-space $x_{4}>0$, then (1.1) cannot hold; (ii) the Sobolev-Poincaré inequality is false in the homogeneous ball $\left(x_{1}^{2}+x_{2}^{2}\right)^{6}+x_{3}^{6}+x_{4}^{4}<1$. The latter fact disproves a conjecture stated in [CG, p. 429]. 
In a forthcoming paper we shall show how conditions similar to (1.2) can be used to identify regular domains in the different context of diagonal vector fields of the form $X_{j}=\lambda_{j}(x) \frac{\partial}{\partial x_{j}}, j=1, \ldots, n$, where the $\lambda_{j}$ 's are suitable functions.

In the setting of step 2 groups the situation is different. It has been proved that Carnot-Carathéodory balls in the Heisenberg group are uniform domains (see [VG] but not NTA domains (see [CG]); examples of uniform domains in the Heisenberg group can also be found by means of quasiconformal mappings (see [CT]); half spaces and cubes centered at the origin in the Heisenberg group are uniform domains (see $\mathrm{G}]$ ). It was conjectured in GN1, $\mathrm{CG}$ and CGN1 that in any group of step 2 a connected, bounded open set with boundary of class $C^{1,1}$ is an NTA domain. A partial positive answer to this conjecture has been given in [CG] for sets with cylindrical symmetry near each characteristic point. An improvement has been announced to the authors by Luca Capogna and Nicola Garofalo for sets with "strongly isolated" characteristic set in the Heisenberg group. In the present paper we prove the full statement:

Theorem 1.3. Any $C^{1,1}$ domain in a step 2 homogeneous group is NTA.

Theorem 1.3 is sharp in the following sense: in groups of step 2, for any $\alpha \in(0,1)$ there are open sets of class $C^{1, \alpha}$ for which the Sobolev-Poincaré inequality (1.1) does not hold (see Example 5.1).

Theorem 1.3 was recently used in cooperation with the results of [DGN] to give a complete characterization (in $C^{2}$ domains) of the trace space for Sobolev functions in step 2 homogeneous groups (see [DGN, Thm. 13.5]).

Since the proofs in the paper are technical we would like to sketch here the key ideas in the simple situation of the Heisenberg group. We shall briefly discuss only the John property, requiring a deeper analysis for the uniform condition. Consider the vector fields $X_{1}=\partial_{x_{1}}-x_{2} \partial_{x_{3}}$ and $X_{2}=\partial_{x_{2}}+x_{1} \partial_{x_{3}}$ in $\mathbb{R}^{3}$. Here $\mathbb{R}^{3}$ is equipped with the group law $x \cdot y=\left(x_{1}+y_{1}, x_{2}+y_{2}, x_{3}+y_{3}+x_{1} y_{2}-x_{2} y_{1}\right)$, with the homogeneous norm $\|x\|=\left(\left(x_{1}^{2}+x_{2}^{2}\right)^{2}+x_{3}^{2}\right)^{1 / 4}$ and with the left invariant distance $d(x, y)=\left\|x^{-1} \cdot y\right\|$. We consider an open set $\Omega=\left\{x_{3}>\varphi\left(x_{1}, x_{2}\right)\right\}$, where $\varphi(0,0)=0$ and $\varphi$ is a smooth function. Since rotations around the $x_{3}$-axis are isometries of the group, there is no loss of generality in requiring $\partial_{x_{1}} \varphi(0,0)=-\nu$ with $\nu \geq 0$, and $\partial_{x_{2}} \varphi(0,0)=0$. We shall explain how to construct a John curve, i.e. a curve satisfying condition (1.3) below, starting from the origin. By left translation, this will also produce John curves starting from any point of the boundary of $\Omega$. Moreover, since the map $\left(x_{1}, x_{2}, x_{3}\right) \mapsto\left(x_{1}, x_{2}, x_{3}+\alpha\right), \alpha \in \mathbb{R}$, is a left translation, the natural John curve starting from any point $\left(x_{1}, x_{2}, \varphi\left(x_{1}, x_{2}\right)+\alpha\right), \alpha \geq 0$, can be consequently obtained.

Our curve $\gamma$ will be constructed by two pieces:

$$
\gamma(t)= \begin{cases}(t, 0,0) & \text { if } 0<t \leq \sigma \nu:=t_{1}, \\ \left(t_{1}, 0, t-t_{1}\right) & \text { if } t \geq t_{1} .\end{cases}
$$

Here $\sigma>0$ is a parameter depending on the given function $\varphi$ (it must not depend on $\nu$ ) and will be fixed during the argument. We note that if $\nu=0$, i.e. $\nabla \varphi(0,0)=$ 0 (the origin is a characteristic point), then $t_{1}=0$, the first piece of the path disappears and we simply have $\gamma(t)=(0,0, t)$; this curve is not rectifiable.

We show that $\gamma$ satisfies the following "John property":

$$
\operatorname{dist}(\gamma(t) ; \partial \Omega) \geq \lambda d(\gamma(t), 0) \text { for all } t>0
$$


for some small constant $\lambda>0$ depending on $\varphi$, but not on $\nu$ (in Section 2 (1.3) is compared with similar conditions involving rectifiable curves). Subtracting from $\varphi$ its linear part we get $\varphi(x)=-\nu x_{1}+\psi\left(x_{1}, x_{2}\right)$ where, in view of the smoothness of $\varphi$, the remainder $\psi$ satisfies for some uniform constant $C_{0}>0$ the quadratic growth estimate

$$
\left|\psi\left(x_{1}, x_{2}\right)\right| \leq C_{0}\left(x_{1}^{2}+x_{2}^{2}\right)
$$

(the constant $C_{0}$ is essentially the Lipschitz constant of $\nabla \varphi$ ). Points of the ball $B(\gamma(t), \lambda d(\gamma(t), 0))=\gamma(t) \cdot B(0, \lambda\|\gamma(t)\|)$ are of the form $\gamma(t) \cdot h$, with $\|h\|<\lambda\|\gamma(t)\|$. Thus (1.3) is equivalent to $\gamma(t) \cdot h \in \Omega$, that is,

$$
(\gamma(t) \cdot h)_{3} \geq \varphi(\gamma(t) \cdot h)
$$

for all $h \in \mathbb{R}^{3},\|h\|<\lambda\|\gamma(t)\|$. By the quadratic estimate for $\psi$, (1.4) is ensured by

$$
(\gamma(t) \cdot h)_{3}+\nu(\gamma(t) \cdot h)_{1} \geq C_{0}\left[(\gamma(t) \cdot h)_{1}^{2}+(\gamma(t) \cdot h)_{2}^{2}\right] .
$$

Inequality (1.5) will be first checked for $t \leq t_{1}=\sigma \nu$. Note that

$$
\gamma(t) \cdot h=(t, 0,0) \cdot\left(h_{1}, h_{2}, h_{3}\right)=\left(t+h_{1}, h_{2}, h_{3}+t h_{2}\right) .
$$

Then (1.5) becomes $h_{3}+t h_{2}+\nu\left(t+h_{1}\right) \geq C_{0}\left[\left(t+h_{1}\right)^{2}+h_{2}^{2}\right]$, that is,

$$
\nu\left(t+h_{1}\right) \geq-h_{3}-t h_{2}+C_{0}\left[\left(t+h_{1}\right)^{2}+h_{2}^{2}\right] .
$$

Since $\|h\| \leq \lambda t$, the terms in the right-hand side can be estimated as: $-h_{3} \leq\left|h_{3}\right| \leq$ $(\lambda t)^{2},-t h_{2} \leq\left|t h_{2}\right| \leq \lambda t^{2}$, and the terms in square brackets can be estimated by $C t^{2}$, with $C$ an absolute constant. Moreover, in the left-hand side we have

$$
\nu\left(t+h_{1}\right) \geq \nu(t-\|h\|) \geq \frac{1}{2} \nu t
$$

as soon as $\lambda \leq 1 / 2$. We ultimately get the inequality

$$
\nu t \geq C_{0} t^{2}
$$

where $C_{0}$ is an absolute constant possibly larger than the ones written above. This inequality holds as soon as $t \leq \sigma \nu$, where $\sigma$ is any positive fixed constant satisfying $\sigma \leq 1 / C_{0}$.

We now check (1.5) for $t \geq t_{1}=\sigma \nu$. Note that

$$
\gamma(t) \cdot h=\left(t_{1}, 0, t-t_{1}\right) \cdot h=\left(t_{1}+h_{1}, h_{2}, t-t_{1}+h_{3}+t_{1} h_{2}\right) .
$$

Then (1.5) becomes $t-t_{1}+h_{3}+t_{1} h_{2}+\nu\left(t_{1}+h_{1}\right) \geq C_{0}\left[\left(t_{1}+h_{1}\right)^{2}+h_{2}^{2}\right]$, that is,

$$
t-t_{1}+\nu\left(t_{1}+h_{1}\right) \geq-h_{3}-t_{1} h_{2}+C_{0}\left[\left(t_{1}+h_{1}\right)^{2}+h_{2}^{2}\right]
$$

This inequality has to be checked for $\|h\| \leq \lambda\|\gamma(t)\|=\lambda\left(t_{1}^{4}+\left(t-t_{1}\right)^{2}\right)^{1 / 4}$, or, letting $a=\left(t-t_{1}\right)^{1 / 2}$, for $\|h\| \leq \lambda\left(t_{1}^{4}+a^{4}\right)^{1 / 4}$. The terms in the right-hand side of (1.7) can be estimated as follows: $-h_{3} \leq\left|h_{3}\right| \leq C \lambda\left(t_{1}^{2}+a^{2}\right)$ and $-t_{1} h_{2} \leq\left|t_{1} h_{2}\right| \leq$ $t_{1}\|h\| \leq C \lambda\left(t_{1}^{2}+t_{1} a\right)$. Moreover,

$$
\left(t_{1}+h_{1}\right)^{2} \leq\left(t_{1}+\lambda\left(t_{1}^{4}+a^{4}\right)^{1 / 4}\right)^{2} \leq\left(C t_{1}+C \lambda a\right)^{2},
$$

while $h_{2}^{2} \leq\|h\|^{2} \leq \lambda^{2}\left(t_{1}^{4}+a^{4}\right)^{1 / 2} \leq C\left(t_{1}^{2}+\lambda^{2} a^{2}\right)$. The left-hand side can be estimated as follows:

$$
\nu\left(t_{1}+h_{1}\right) \geq \nu t_{1}-\nu\|h\| \geq \nu t_{1}-\nu \lambda C\left(t_{1}+a\right) \geq \frac{1}{2} \nu t_{1}-\lambda C \nu a,
$$

as soon as $\lambda \leq \frac{1}{2 C}$. 
Then (1.7) will be proved if the following inequality holds:

$$
a^{2}+\frac{1}{2} \nu t_{1}-\lambda C \nu a \geq C \lambda t_{1}^{2}+C \lambda t_{1} a+C \lambda a^{2}+C t_{1}^{2}+C \lambda^{2} a^{2} .
$$

Choosing $\lambda$ small enough the terms $C \lambda a^{2}$ and $C \lambda^{2} a^{2}$ in the right-hand side can be absorbed in the left-hand side. Note also that $C \lambda t_{1} a \leq \frac{C}{2} \lambda t_{1}^{2}+\frac{C}{2} \lambda a^{2}$, so that $\frac{C}{2} \lambda a^{2}$ can be absorbed in the left-hand side, also. Then we find the stronger inequality $a^{2}+\nu t_{1} \geq C\left(t_{1}^{2}+\lambda \nu a+\lambda t_{1} a\right)$ which is ensured by $a^{2}+\nu t_{1} \geq C\left(t_{1}^{2}+\lambda \nu a\right)$, and recalling now that $t_{1}=\sigma \nu$ we finally get

$$
a^{2}+\sigma \nu^{2} \geq C_{1}\left(\sigma^{2} \nu^{2}+\lambda \nu a\right) .
$$

We slightly modify the choice of $\sigma$ requiring $C_{1} \sigma^{2} \leq \sigma / 2$. Then, in order to prove (1.8) it is enough to verify that

$$
a^{2}+\frac{1}{2} \sigma \nu^{2} \geq C_{1} \lambda\left(\frac{1}{2} \nu^{2}+\frac{1}{2} a^{2}\right) .
$$

This inequality is satisfied for any $a \geq 0$ provided that $C_{1} \lambda / 2 \leq 1$ and $C_{1} \lambda \leq \sigma$. Indeed, such a $\lambda$ can be found and it does not depend on $\nu$, as required.

The described argument proves that smooth domains in the Heisenberg group enjoy the John property. They also enjoy the uniform property; this can be proved taking suitable cones having as core the John curves constructed above and showing that these cones meet appropriately (see Theorem 3.2).

Before closing this introduction we briefly explain our notation. In the paper we denote by $C$ (or $k$ ) absolute positive constants (they may depend on the surface we are considering). By $a \lesssim b$ we mean $a \leq C b$, and by $a \simeq b$ we mean $C^{-1} a \leq b \leq C a$. By $\varepsilon_{0}$ and $C_{0}$ we denote, as well, absolute positive constants which are respectively smaller and larger than 1 . The small parameter $\lambda$ is used to denote the "aperture" of cones and in Sections 3 and 4 we shall several times write $\lambda$ instead of $o(1)$, as $\lambda \rightarrow 0$. Finally, if $x, y \in \mathbb{R}^{n}$, then $\langle x, y\rangle=\sum_{i=1}^{n} x_{i} y_{i}$ denotes the usual Euclidean inner product.

\section{John, Uniform And NTA DOMAIns. Some general faCts}

In this section we recall the basic definitions and some general known results concerning John, uniform and NTA domains (see, for example, [MS, [V], [CT], CG], HK] ) We consider a metric space $(M, d)$. If $\gamma:[0,1] \rightarrow M$ is a curve, we denote by length $(\gamma)$ the length of $\gamma$ and by $\gamma_{[a, b]}:[0, b-a] \rightarrow M$ the curve $\gamma_{[a, b]}(t)=\gamma(t+a), 0 \leq a \leq b \leq 1$. A curve is rectifiable if length $(\gamma)<+\infty$.

Definition 2.1. Let $(M, d)$ be a metric space. A bounded open set $\Omega \subset M$ is a John domain if there exist $x_{0} \in \Omega$ and $\lambda>0$ such that for every $x \in \Omega$ there exists a continuous rectifiable curve $\gamma:[0,1] \rightarrow \Omega$, such that $\gamma(0)=x, \gamma(1)=x_{0}$ and

$$
\operatorname{dist}(\gamma(t) ; \partial \Omega) \geq \lambda \operatorname{length}\left(\gamma_{[0, t]}\right)
$$

for all $t \in[0,1]$.

Definition 2.2. Let $(M, d)$ be a metric space. An open set $\Omega \subset M$ is a uniform domain if there exists $\lambda>0$ such that for every $x, y \in \Omega$ there exists a continuous rectifiable curve $\gamma:[0,1] \rightarrow \Omega$ connecting them and such that

$$
\operatorname{length}(\gamma) \leq \frac{1}{\lambda} d(x, y)
$$


and

$$
\operatorname{dist}(\gamma(t) ; \partial \Omega) \geq \lambda \min \left\{\operatorname{length}\left(\gamma_{[0, t]}\right), \operatorname{length}\left(\gamma_{[t, 1]}\right)\right\}
$$

for all $t \in[0,1]$.

Let $\Omega$ be an open set in the metric space $M$ and let $k \geq 1$. A ball $B(x, r) \subset \Omega$ is $k$-non-tangential if $k^{-1} r \leq \operatorname{dist}(B ; \partial \Omega) \leq k r$. Given $x, y \in \Omega$, a Harnack chain joining $x$ to $y$ is a family $B_{1}, \ldots, B_{n}$ of $k$-non-tangential balls in $\Omega$ (for some $k \geq 1$ ) such that $x \in B_{1}, y \in B_{n}$ and $B_{j} \cap B_{j+1} \neq \varnothing$. The integer $n$ is the length of the chain.

Definition 2.3. Let $(M, d)$ be a metric space. A bounded domain $\Omega \subset M$ is non-tangentially accessible if there exist $r_{0}>0$ and $k \geq 1$ such that:

(i) Corkskrew condition. For all $x \in \partial \Omega$ and $r \in\left(0, r_{0}\right)$ there exists $y \in \Omega$ such that $k^{-1} r \leq d(x, y) \leq r$ and $\operatorname{dist}(y ; \partial \Omega) \geq k^{-1} r$.

(ii) The set $M \backslash \bar{\Omega}$ satisfies the corkscrew condition.

(iii) Harnack chain condition. If $\epsilon>0$ and $x, y \in \Omega$ with $\operatorname{dist}(x ; \partial \Omega)>\epsilon$, $\operatorname{dist}(y ; \partial \Omega)>\epsilon$ and $d(x, y)<C \epsilon$, then there exists a Harnack chain of $k$ non-tangential balls joining $x$ to $y$ with length depending on $C$ but not on $\epsilon$.

This paper deals with homogeneous groups. Endowed with their Carnot-Carathéodory distance homogeneous groups are metric spaces with geodesics, and moreover, if $\mu$ denotes their Haar measure (which is Lebesgue measure), then there are positive constants $C$ and $Q \in \mathbb{N}$ such that $\mu(B(x, r))=C r^{Q}$ for all $x$ belonging to the group and for all $r>0$ (see $[\mathrm{FS}$ ). More generally, a metric space $(M, d)$ endowed with a Borel measure $\mu$ such that $0<\mu(B(x, 2 r)) \leq \delta \mu(B(x, r))<+\infty$ for all $x \in M$ and $r>0$ is called a doubling metric space, and $\delta>0$ is its doubling constant.

We establish some propositions that will be needed in the next sections.

Proposition 2.4. Let $(M, d)$ be a doubling metric space with geodesics. Let $\Omega \subset M$ be a bounded open set and for any $r>0$ define $\Omega_{r}=\{y \in \Omega$ : $\operatorname{dist}(y ; \partial \Omega)>r\}$. Assume that there exist $r>0$ and $\lambda>0$ such that $\Omega_{r}$ is arcwise connected and such that for any $x \in \Omega$ there exists a continuous curve $\gamma_{x}:[0,1] \rightarrow \Omega$ such that $\gamma_{x}(0)=x, \gamma_{x}(1) \in \Omega_{r}$ and

$$
\operatorname{dist}\left(\gamma_{x}(t) ; \partial \Omega\right) \geq \lambda d\left(\gamma_{x}(t), x\right)
$$

for all $t \in[0,1]$. Then $\Omega$ is a John domain.

Proof. We show that there exist $x_{0} \in \Omega$ and $C>0$ such that for all $x \in \Omega$ there exists a continuous curve $\gamma:[0,1] \rightarrow \Omega$ such that $\gamma(0)=x, \gamma(1)=x_{0}$ and (2.3) holds. In the terminology of [HK] this means that $\Omega$ is a weak John domain. Since $(M, d)$ is a doubling metric space with geodesics from [HK, Proposition 9.6], it follows that $\Omega$ is a John domain.

Fix $x_{0} \in \Omega_{r}$ and for any $y \in \Omega_{r}$ denote by $\gamma_{y, x_{0}}:[0,1] \rightarrow \Omega_{r}$ a continuous path such that $\gamma_{y, x_{0}}(0)=y$ and $\gamma_{y, x_{0}}(1)=x_{0}$. Then $\operatorname{dist}\left(\gamma_{y, x_{0}}(t) ; \partial \Omega\right) \geq r$ and $d\left(\gamma_{y, x_{0}}(t), x\right) \leq \operatorname{diam}(\Omega)$ for all $t \in[0,1]$. If $x \in \Omega$, let $\gamma_{x}$ be as in the statement of the proposition, let $\bar{y}=\gamma_{x}(1)$ and consider $\gamma_{\bar{y}, x_{0}}$. The path $\gamma$ sum of $\gamma_{x}$ and $\gamma_{\bar{y}, x_{0}}$ enjoys property (2.3) (possibly with a new $\lambda$ ). 
Proposition 2.5. Let $(M, d)$ be a doubling metric space with geodesics. Let $\Omega \subset M$ be a bounded open set. If there exist $\lambda>0$ and $r>0$ such that for any $z \in \partial \Omega$ and for all $x, y \in B(z, r) \cap \Omega$ there exists a continuous curve $\gamma:[0,1] \rightarrow \Omega$ such that $\gamma(0)=x, \gamma(1)=y$,

$$
\operatorname{diam}(\gamma) \leq \frac{1}{\lambda} d(x, y)
$$

and

$$
\operatorname{dist}(\gamma(t) ; \partial \Omega) \geq \lambda \min \left\{\operatorname{diam}\left(\gamma_{[0, t]}\right), \operatorname{diam}\left(\gamma_{[t, 1]}\right)\right\}
$$

for all $t \in[0,1]$, then $\Omega$ is an uniform domain.

Proof. The proof relies on two facts. First, using the argument in [V], Theorem 4.1], which holds in any metric space, it can be proved that for all $x, y \in \Omega$ there is a continuous curve $\gamma$ such that (2.4) and (2.5) hold (the constant $\lambda$ may be different).

Second, curves can be taken rectifiable and satisfying (2.1) and (2.2). We sketch the proof. Let $x, y \in \Omega$ and fix a continuous curve $\gamma$ such that $\gamma(0)=x$ and $\gamma(1)=$ $y$, and such that (2.4) and (2.5) hold. Let $\bar{t} \in(0,1)$ be such that $\operatorname{diam}\left(\gamma_{[0, \bar{t}}\right)=$ $\operatorname{diam}\left(\gamma_{[\bar{t}, 1]}\right)$. Consider the path $\tilde{\gamma}_{x}=\gamma_{[0, \bar{t}]}$. By the choice of $\bar{t}$ and by (2.5) $\tilde{\gamma}_{x}$ $\operatorname{satisfies} \operatorname{dist}\left(\tilde{\gamma}_{x}(t) ; \partial \Omega\right) \geq \lambda \operatorname{diam}\left(\left(\tilde{\gamma}_{x}\right)_{[0, t]}\right)$. By Lemma 2.7 in [MS], which can be proved in any doubling metric space with geodesics, there exists a rectifiable path $\gamma_{x}$ such that $\gamma_{x}(0)=x, \gamma_{x}(1)=\gamma(\bar{t})$ and $\operatorname{dist}\left(\gamma_{x}(t) ; \partial \Omega\right) \geq \lambda^{\prime} \operatorname{length}\left(\gamma_{[0, t]}\right)$ (here $\lambda^{\prime}$ depends on $\lambda$ and on the doubling constant). A rectifiable path $\gamma_{y}$ can be analogously constructed. The sum of the paths $\gamma_{x}$ and $\gamma_{y}$ gives the required path and proves that $\Omega$ is actually a uniform domain in the sense of Definition 2.2

A continuous curve $\gamma:[0,1] \rightarrow \Omega$ such that $\gamma(0)=x$ and $\operatorname{dist}(\gamma(t) ; \partial \Omega) \geq$ $\lambda d(\gamma(t), x)$ will be called a John curve starting from $x$ and with constant $\lambda>0$.

All the John curves constructed in Sections 3 and 4 are such that the function $t \mapsto d(\gamma(t), \gamma(0))$ is (equivalent to) a monotonic increasing function and satisfies

$$
d(\gamma(0), \gamma(t)) \simeq \operatorname{diam}\left(\gamma_{[0, t]}\right)
$$

In Section 3 we shall refer to the following proposition.

Proposition 2.6. Let $(M, d)$ be a doubling metric space with geodesics and let $\Omega \subset M$ be a bounded open set. Assume that there exists $\lambda>0$ and $r>0$ such that for any $z \in \partial \Omega$ and for all $x, y \in B(z, r) \cap \Omega$ there exist two John curves $\gamma_{x}, \gamma_{y}:[0,1] \rightarrow \Omega$ starting respectively from $x$ and $y$, with John constant $\lambda$, such that $\gamma_{x}(1)=\gamma_{y}(1)$ and

$$
\max \left\{\operatorname{diam}\left(\gamma_{x}\right), \operatorname{diam}\left(\gamma_{y}\right)\right\} \leq \frac{1}{\lambda} d(x, y) .
$$

Assume also that $\gamma_{x}$ and $\gamma_{y}$ verify (2.6). Then $\Omega$ is a uniform domain.

Proof. Let $\gamma$ be the curve sum of $\gamma_{x}$ and $\gamma_{y}$ parameterized over [0,2]. First of all

$$
\operatorname{diam}(\gamma) \leq \operatorname{diam}\left(\gamma_{x}\right)+\operatorname{diam}\left(\gamma_{y}\right) \leq \frac{2}{\lambda} d(x, y) .
$$

Consider now a point $\gamma(t)$ and assume that $\gamma(t)=\gamma_{x}(t)$. Then

$$
\begin{aligned}
\operatorname{dist}(\gamma(t) ; \partial \Omega) & =\operatorname{dist}\left(\gamma_{x}(t) ; \partial \Omega\right) \geq \lambda d\left(\gamma_{x}(t), x\right) \\
& \simeq \lambda \operatorname{diam}\left(\left(\gamma_{x}\right)_{[0, t]}\right) \geq \lambda \min \left\{\operatorname{diam}\left(\gamma_{[0, t]}\right), \operatorname{diam}\left(\gamma_{[t, 2]}\right)\right\} .
\end{aligned}
$$

If $\gamma(t)$ is in $\gamma_{y}$, the estimate is the same. The claim follows from Proposition 2.5. 
It is easy to see that if both $\Omega$ and $M \backslash \Omega$ satisfy the hypotheses of Proposition 2.6 then the interior and the exterior corkskrew conditions are satisfied. The following result contains another useful connection between uniform and NTA domains.

Proposition 2.7. Let $(M, d)$ be a metric space. If $\Omega \subset M$ is a uniform domain, then $\Omega$ satisfies the Harnack chain condition (iii) in Definition 2.3

Proof. See [CT, Proposition 4.2]

\section{UNIFORM AND NTA DOMAINS IN GROUPS OF STEP 2}

In this section we study uniform and NTA domains in homogeneous groups of step 2. We shall work in $\mathbb{R}^{n}$ endowed a left invariant metric induced by a system of vector fields $X=\left(X_{1}, \ldots, X_{m}\right)$ which generates a stratified Lie algebra of step 2 . In $\mathbb{R}^{n}=\mathbb{R}^{m} \times \mathbb{R}^{q}$ we denote $x=\left(x^{\prime}, x^{\prime \prime}\right) \in \mathbb{R}^{m} \times \mathbb{R}^{q}$ and by abuse of notation we shall write $x^{\prime}=\left(x^{\prime}, 0\right)$ and $x^{\prime \prime}=\left(0, x^{\prime \prime}\right)$. We say that $x^{\prime}$ are the variables of the first slice and that $x^{\prime \prime}$ are the variables of the second slice.

The vector fields can be assumed to be of the form

$$
X_{j}=\partial_{j}+\sum_{k=m+1}^{n} q_{j k} \partial_{k}, \quad j=1, \ldots, m,
$$

where $q_{j k}=q_{j k}\left(x^{\prime}\right)$ are homogeneous polynomials of degree 1 in the variables $x^{\prime}$ (see [FS]). Introduce the group law

$$
\begin{aligned}
x \cdot y=x+y+Q(x, y)=\left(x_{1}+y_{1}\right. & , \ldots, x_{m}+y_{m}, x_{m+1}+y_{m+1} \\
& \left.+Q_{m+1}(x, y), \ldots, x_{n}+y_{n}+Q_{n}(x, y)\right),
\end{aligned}
$$

where $Q=\left(Q_{1}, \ldots, Q_{n}\right)$ with $Q_{1}=\ldots=Q_{m}=0$, and $Q_{j}=Q_{j}\left(x^{\prime}, y^{\prime}\right), j=$ $m+1, \ldots, n$, are homogeneous polynomials of degree 2 (the fact that the $Q_{j}$ 's do not depend on the variables of the second slice will be used several times). Moreover, they can be assumed to satisfy

$$
\left|Q_{j}\left(x^{\prime}, y^{\prime}\right)\right| \leq C\left|x^{\prime}\right|\left|y^{\prime}\right|
$$

The vector fields $X_{1}, \ldots, X_{m}$ are by assumption left invariant with respect to the introduced law.

We denote by $d$ the Carnot-Carathéodory distance induced on $\mathbb{R}^{n}$ by $X_{1}, \ldots, X_{m}$ and by $B(x, r)$ the open ball centered at $x \in \mathbb{R}^{n}$ with radius $r \geq 0$. We also introduce in $\mathbb{R}^{n}$ the following continuous homogeneous norm

$$
\|x\|=\left|x^{\prime}\right|+\left|x^{\prime \prime}\right|^{1 / 2} \text {. }
$$

By a standard argument it can be proved that $d(x, y) \simeq\left\|y^{-1} \cdot x\right\|$. Thus, letting $\operatorname{Box}(x, r)=\left\{x \cdot y \in \mathbb{R}^{n}:\|y\| \leq r\right\}$ there exists $c>1$ such that for all $x \in \mathbb{R}^{n}$ and $r \geq 0$

$$
\operatorname{Box}\left(x, c^{-1} r\right) \subset B(x, r) \subset \operatorname{Box}(x, c r) .
$$

Definition 3.1. Let $S \subset \mathbb{R}^{n}$ be a hypersurface of class $C^{1}$ given in a neighborhood $\mathcal{U}$ of $x_{0} \in S$ by the local equation $\Phi=0$ where $\Phi \in C^{1}(\mathcal{U})$. The point $x_{0}$ is characteristic if $X_{1} \Phi\left(x_{0}\right)=\ldots=X_{m} \Phi\left(x_{0}\right)=0$.

We denote by $e_{j}$ the $j$ th coordinate versor and if $x=\sum_{i=1}^{n} x_{i} e_{i} \in \mathbb{R}^{n}$ and $j \in\{1, \ldots, n\}$ we let

$$
\hat{x}_{j}=\sum_{1 \leq i \leq n, i \neq j} x_{i} e_{i}
$$


Let $\Omega \subset \mathbb{R}^{n}$ be an open set, $x_{0} \in \partial \Omega$ and let $\mathcal{U} \subset \mathbb{R}^{n}$ be a neighborhood of $x_{0}$ such that $\partial \Omega \cap \mathcal{U}=\{x \in \mathcal{U}: \Phi(x)=0\}$ for some defining function $\Phi \in C^{1}(\mathcal{U})$. If $x \in \partial \Omega \cap \mathcal{U}$, then, possibly shrinking $\mathcal{U}$, the translated surface $x^{-1} \cdot(\partial \Omega \cap \mathcal{U})$ can be expressed in parametric form by an equation of the type $y_{j}=\varphi\left(\hat{y}_{j}\right)$ for $\hat{y}_{j}$ belonging to a neighborhood of the origin in $\mathbb{R}^{n-1}$ and $\varphi$ of class $C^{1}$. If $x_{0}$ is a characteristic point we must choose $j \in\{m+1, \ldots, n\}$, otherwise we can choose $j \in\{1, \ldots, m\}$. In the next theorem we shall assume $\Phi \in C^{1,1}$ and so $\varphi \in C^{1,1}$. Actually, in the proof we shall need the Lipschitz continuity only of the derivatives $\partial_{1} \varphi, \ldots, \partial_{m} \varphi$.

Theorem 3.2. Any connected, bounded open set $\Omega \subset \mathbb{R}^{n}$ of class $C^{1,1}$ is an NTA domain in the metric space $\left(\mathbb{R}^{n}, d\right)$.

Proof. The proof will be split into several numbered small steps.

1. We claim that for all $x_{0} \in \partial \Omega$ there exists a neighborhood $\mathcal{U}$ of $x_{0}$ such that for all $x, y \in \mathcal{U} \cap \Omega$ there exist continuous curves $\gamma_{x}$ and $\gamma_{y}:[0,1] \rightarrow \Omega$ satisfying the hypotheses of Proposition 2.6. The proof will show that conditions (i) and (ii) in Definition [2.3 are verified, and by Proposition [2.7 $\Omega$ will be an NTA domain.

2. Let $\mathcal{U}$ be a neighborhood of $x_{0}$ and let $\Phi \in C^{1}(\mathcal{U})$ be a defining function such that $\partial \Omega \cap \mathcal{U}=\{x \in \mathcal{U}: \Phi(x)=0\}$. We shall distinguish two cases:

(C1) $\left|X_{1} \Phi\left(x_{0}\right)\right|=\ldots=\left|X_{m} \Phi\left(x_{0}\right)\right|=0\left(x_{0}\right.$ is a characteristic point of $\left.\partial \Omega\right)$;

(C2) $\left|X_{1} \Phi\left(x_{0}\right)\right|+\ldots+\left|X_{m} \Phi\left(x_{0}\right)\right|>0$ ( $x_{0}$ is a non-characteristic point of $\partial \Omega$ ).

Let $x_{j}=\varphi\left(\hat{x}_{j}\right)$ be a local parameterization of $\partial \Omega \cap \mathcal{U}$ around $x_{0}$. We assume without loss of generality that $x_{0}=0$.

3. Case 1. We consider an open set $\left\{y \in \mathbb{R}^{n}: y_{j}>\varphi\left(\hat{y}_{j}\right)\right\}$ where $j>m$ and $\varphi \in C^{1}\left(\mathbb{R}^{n-1}\right)$ is a function such that $\varphi(0)=0,|\nabla \varphi| \leq k$ and $\partial_{1} \varphi, \ldots, \partial_{m} \varphi$ are $k$-Lipschitz continuous functions, where $k>0$ is a fixed given constant. Define

$$
\nu_{i}=-\partial_{i} \varphi(0), \quad \text { for } i=1, \ldots, m, \quad \text { and } \quad \nu=\left(\nu_{1}, \ldots, \nu_{m}, 0, \ldots, 0\right) .
$$

Write also

$$
\varphi\left(\hat{y}_{j}\right)=-\sum_{i=1}^{m} \nu_{i} y_{i}+\psi\left(\hat{y}_{j}\right)
$$

where $\psi$ can be written by Taylor formula in the form

$$
\psi\left(\hat{y}_{j}\right)=\varphi\left(\hat{y}_{j}\right)-\sum_{i=1}^{m} \partial_{i} \varphi(0) y_{j}=\sum_{i>m, i \neq j} \partial_{i} \varphi(0) y_{i}+O\left(\left|\hat{y}_{j}\right|^{2}\right),
$$

and satisfies the growth estimate

$$
\left|\psi\left(\hat{y}_{j}\right)\right| \lesssim\left\|\hat{y}_{j}\right\|^{2}
$$

Here we used the homogeneous norm introduced in (3.3) and the Lipschitz continuity of $\partial_{1} \varphi, \ldots, \partial_{m} \varphi$.

Our construction will take place in two steps. In the first step we define "canonical" John curves starting from points near the boundary. In the second step we join points near the boundary by curves satisfying the hypotheses of Proposition 2.6 .

4. First step. Define

$$
N_{1}=\frac{\nu_{1}}{|\nu|}, \ldots, N_{m}=\frac{\nu_{m}}{|\nu|} \quad \text { and } \quad N=\left(N_{1}, \ldots, N_{m}, 0, \ldots, 0\right),
$$


and if $\nu=0$, simply set $N=0$. For $\sigma>0$ let $t_{1}=\sigma|\nu|$. Fix $x=x_{j} e_{j}$ with $x_{j} \geq 0$ and define the continuous curve $\gamma:[0,1] \rightarrow \mathbb{R}^{n}$,

$$
\gamma(t)= \begin{cases}x \cdot t N=t N+x_{j} e_{j}, & \text { if } 0 \leq t \leq t_{1} \\ x \cdot\left(\left(t_{1} N\right)+\left(t-t_{1}\right) e_{j}\right)=t_{1} N+\left(t-t_{1}+x_{j}\right) e_{j}, & \text { if } t_{1} \leq t \leq 1\end{cases}
$$

5. We claim that there exist $\sigma, \lambda \in(0,1)$ such that for all $t \in[0,1]$,

$$
\operatorname{dist}(\gamma(t) ; \partial \Omega) \geq \lambda d(\gamma(t), x) .
$$

If $0 \leq t \leq t_{1}$, then $d(\gamma(t), x) \simeq\left\|x^{-1} \cdot \gamma(t)\right\|=\|t N\|=t$, and (3.6) is equivalent to

$$
\operatorname{Box}(\gamma(t), \lambda t) \cap\left\{y_{j}=\varphi\left(\hat{y}_{j}\right)\right\}=\varnothing,
$$

which is implied by

$$
\langle\nu, y\rangle+y_{j} \geq\left|\psi\left(\hat{y}_{j}\right)\right|, \quad \text { for all } y \in \operatorname{Box}(\gamma(t), \lambda t) .
$$

Points in $\operatorname{Box}(\gamma(t), \lambda t)$ are of the form

$$
\gamma(t) \cdot h=\left(t N+x_{j} e_{j}\right) \cdot h=t N+x_{j} e_{j}+h+Q(t N, h)
$$

with $\|h\| \leq \lambda t$ ( $Q$ does not depend on the variables on the second slice) and thus we have to check that

$$
\langle\nu, t N+h\rangle+x_{j}+h_{j}+Q_{j}(t N, h) \geq\left|\psi\left((\widehat{(t) \cdot h})_{j}\right)\right|
$$

which is guaranteed by

$$
t|\nu|+\langle\nu, h\rangle+x_{j} \geq\left|h_{j}\right|+\left|Q_{j}(t N, h)\right|+\left|\psi\left((\gamma \widehat{(t) \cdot h})_{j}\right)\right| .
$$

Now, since $|\langle\nu, h\rangle| \leq \lambda|\nu| t$, then $t|\nu|+\langle\nu, h\rangle \gtrsim t|\nu|$ as soon as $\lambda<1 / 2$. Moreover, $\left|h_{j}\right| \leq t^{2}$ and by (3.4)

$$
\begin{aligned}
\left|\psi\left((\widehat{\gamma(t) \cdot h})_{j}\right)\right| & \lesssim\left\|(\widehat{(t) \cdot h})_{j}\right\|^{2}=\left\|t N+\hat{h}_{j}+\hat{Q}_{j}(t N, h)\right\|^{2} \\
& \lesssim t^{2}+\|h\|^{2}+\|Q(t N, h)\|^{2} \lesssim t^{2} .
\end{aligned}
$$

Moreover, $\left|Q_{j}(t N, h)\right| \lesssim \lambda t^{2} \lesssim t^{2}$. Thus (3.7) is implied by

$$
\epsilon_{0}\left(t|\nu|+x_{j}\right) \geq t^{2}
$$

where $\epsilon_{0}$ is a small but absolute constant. Since $x_{j} \geq 0$, (3.10) holds for all $t \leq \sigma|\nu|$ as soon as $\sigma \leq \epsilon_{0}$. Our claim is proved if $0 \leq t \leq t_{1}$.

6 . We study the case $t \geq t_{1}$. Notice that in this case

$$
d(\gamma(t), x) \simeq\left\|x^{-1} \cdot \gamma(t)\right\| \simeq t_{1}+\left(t-t_{1}\right)^{1 / 2}=: \delta(t) .
$$

Let $a=\left(t-t_{1}\right)^{1 / 2}$ so that $\delta(t)=t_{1}+a$. We shall sometimes write $\delta$ instead of $\delta(t)$. We claim that there exists $\lambda \in(0,1)$ such that the John property $\operatorname{Box}(\gamma(t), \lambda \delta(t)) \cap$ $\left\{y_{j}=\varphi\left(\hat{y}_{j}\right)\right\}=\varnothing$ holds for all $t \geq t_{1}$.

Points in $\operatorname{Box}(\gamma(t), \lambda \delta)$ are of the form

$$
\begin{aligned}
\gamma(t) \cdot h & =\left(t_{1} N+\left(t-t_{1}+x_{j}\right) e_{j}\right) \cdot h \\
& =t_{1} N+\left(t-t_{1}+x_{j}\right) e_{j}+h+Q\left(t_{1} N, h\right),
\end{aligned}
$$

with $\|h\| \leq \lambda \delta$. Thus, the John property is ensured by

$$
\left\langle\nu, t_{1} N+h\right\rangle+\left(t-t_{1}\right)+x_{j}+Q_{j}\left(t_{1} N, h\right) \geq\left|\psi\left(t_{1} N+\hat{h}_{j}+\hat{Q}_{j}\left(t_{1} N, h\right)\right)\right|,
$$

which (let $t-t_{1}=a^{2}$ ) is a consequence of the following stronger inequality:

$$
t_{1}|\nu|+a^{2}+x_{j} \geq|\nu||| h|+| Q_{j}\left(t_{1} N, h\right)|+| \psi(z) \mid,
$$

where $z$ denotes the argument of $\psi$ in the previous inequality. 
Now, $|\nu|\|h\| \lesssim \lambda|\nu| t_{1}+\lambda|\nu| a$ and $\lambda|\nu| t_{1}$ can be absorbed in the left-hand side of (3.13) as soon as $\lambda \leq \frac{1}{2}$. We also note that $\left|Q_{j}\left(t_{1} N, h\right)\right| \leq t_{1}\|h\| \leq t_{1} \lambda \delta \leq t_{1}^{2}+\lambda \delta^{2}$. Moreover,

$$
\|z\| \lesssim t_{1}+\|h\|+\left\|\hat{Q}_{j}\left(t_{1} N, h\right)\right\| \lesssim t_{1}+\lambda \delta+\left(t_{1} \lambda \delta\right)^{1 / 2} \simeq t_{1}+\lambda \delta,
$$

and by (3.4)

$$
|\psi(z)| \lesssim\|z\|^{2} \lesssim t_{1}^{2}+\lambda \delta^{2}+\lambda t_{1} \delta \simeq t_{1}^{2}+\lambda \delta^{2} \simeq t_{1}^{2}+\lambda a^{2} .
$$

Since the term $\lambda a^{2}$ can be absorbed in the left-hand side and $x_{j} \geq 0$, then (3.13) will follow if we prove that for all $a \geq 0$ it is $\epsilon_{0}\left(t_{1}|\nu|+a^{2}\right) \geq t_{1}^{2}+\lambda|\nu| a$ where $\epsilon_{0}>0$ is a small but absolute constant. Replacing $t_{1}=\sigma|\nu|$ we get

$$
\epsilon_{0}\left(\sigma|\nu|^{2}+a^{2}\right) \geq \sigma^{2}|\nu|^{2}+\lambda|\nu| a .
$$

Now, since $\sigma^{2}|\nu|^{2}+\lambda|\nu| a \leq\left(\sigma^{2}+\lambda / 2\right)|\nu|^{2}+\lambda a^{2} / 2$, (3.14) holds for all $a \geq 0$ provided $\sigma^{2}+\lambda / 2<\epsilon_{0} \sigma$ and $\lambda / 2 \leq \epsilon_{0}$.

7. Second step. We prove that, given $x$ and $y$ in the open set $\left\{z_{j}>\varphi\left(\hat{z}_{j}\right)\right\}$ there exists a continuous curve connecting them and satisfying (2.4) and (2.5). Without loss of generality, we can assume that $x=x_{j} e_{j}$ with $x_{j} \geq 0$ and $y=y_{j} e_{j}+\hat{y}_{j}$ with $y_{j}>\varphi\left(\hat{y}_{j}\right)$. In the first step (see (3.5)) the "canonical" John curve starting from $x$ has been defined. The parameters $\nu, N$ and $t_{1}=\sigma|\nu|$ are defined as in the first step and are relative to $x$. The constant $\sigma$ does not depend on $x$.

8. Our next task is to define the curve starting from $y$. We first explain the "intrinsic" argument which provides the parameters $\nu_{j}$ 's relative to $x$. Letting $\Phi(\xi)=\xi_{j}-\varphi\left(\hat{\xi}_{j}\right)$, we have for $i=1, \ldots, m$,

$$
X_{i} \Phi(\xi)=-\partial_{i} \varphi\left(\hat{\xi}_{j}\right)+\sum_{k>m} q_{i k}(\xi) \partial_{k} \Phi(\xi),
$$

and hence

$$
\nu_{i}=-\partial_{i} \varphi(0)=X_{i} \Phi(0) .
$$

Now let $w=\hat{y}_{j}+\varphi\left(\hat{y}_{j}\right) e_{j}$. We look for the parameters $\nu_{i}, i=1, \ldots, m$, of the curve starting from $w^{-1} \cdot y=\left(y_{j}-\varphi\left(\hat{y}_{j}\right)\right) e_{j}$ relatively to the translated boundary $w^{-1} \cdot\left\{z_{j}=\varphi\left(\hat{z}_{j}\right)\right\}$. Denote these parameters by $\bar{\nu}_{1}, \ldots, \bar{\nu}_{m}$. Then we find by left invariance

$$
\begin{aligned}
\bar{\nu}_{i} & =\left(X_{i} \Phi\right)\left(\hat{y}_{j}+\varphi\left(\hat{y}_{j}\right) e_{j}\right)=-\partial_{i} \varphi\left(\hat{y}_{j}\right)+\left.\sum_{k>m} q_{i k}(y) \frac{\partial}{\partial \xi_{k}}\left(\xi_{j}-\varphi\left(\hat{\xi}_{j}\right)\right)\right|_{\hat{\xi}_{j}=\hat{y}_{j}, \xi_{j}=\varphi\left(\hat{y}_{j}\right)} \\
& =-\partial_{i} \varphi\left(\hat{y}_{j}\right)+q_{i j}\left(y^{\prime}\right)-\sum_{k>m, k \neq j} q_{i k}(y) \partial_{k} \varphi\left(\hat{y}_{j}\right) .
\end{aligned}
$$

Define

$$
\bar{N}=\left(\frac{\bar{\nu}_{1}}{|\bar{\nu}|}, \ldots, \frac{\bar{\nu}_{m}}{|\bar{\nu}|}, 0, \ldots, 0\right) \quad \text { and } \quad \bar{t}_{1}=\sigma|\bar{\nu}| .
$$

The "canonical" John curve $\gamma_{y}$ starting from $y$ can be defined (by left translation of (3.5)) in the following way. If $0 \leq t \leq \bar{t}_{1}$, let

$$
\begin{aligned}
\gamma_{y}(t) & =\left(\hat{y}_{j}+\varphi\left(\hat{y}_{j}\right) e_{j}\right) \cdot\left(t \bar{N}+\left(y_{j}-\varphi\left(\hat{y}_{j}\right)\right) e_{j}\right) \\
& =\hat{y}_{j}+t \bar{N}+y_{j} e_{j}+Q(y, t \bar{N}),
\end{aligned}
$$


and if $t \geq \bar{t}_{1}$, let

$$
\begin{aligned}
\gamma_{y}(t) & =\left(\hat{y}_{j}+\varphi\left(\hat{y}_{j}\right) e_{j}\right) \cdot\left(\bar{t}_{1} \bar{N}+\left(t-\bar{t}_{1}+\left(y_{j}-\varphi\left(\hat{y}_{j}\right)\right) e_{j}\right)\right. \\
& =\hat{y}_{j}+\bar{t}_{1} \bar{N}+\left(t-\bar{t}_{1}+y_{j}\right) e_{j}+Q\left(y, \bar{t}_{1} \bar{N}\right) .
\end{aligned}
$$

9. Denote by $\gamma_{x}$ and $\gamma_{y}$ the curves starting from $x$ and $y$. The curves $\gamma_{x}$ and $\gamma_{y}$ cannot be expected to meet as Proposition 2.6 requires. Thus we enlarge the curve $\gamma_{x}$ by constructing a curvilinear cone around it. Define

$$
\delta(t)= \begin{cases}t & \text { if } 0 \leq t \leq t_{1}, \\ t_{1}+\left(t-t_{1}\right)^{1 / 2} & \text { if } t \geq t_{1},\end{cases}
$$

and recall that $\delta(t) \simeq d\left(\gamma_{x}(t), x\right)$. For $\lambda>0$ let $\mathcal{U}(\lambda)=\left\{h \in \mathbb{R}^{n}:\|h\| \leq \lambda\right\}$, and if $h=\left(h^{\prime}, h^{\prime \prime}\right) \in \mathcal{U}(\lambda)$, define $h_{t}=\left(\delta(t) h^{\prime}, \delta(t)^{2} h^{\prime \prime}\right)$. As $h \in \mathcal{U}(\lambda)$, the family of curves

$$
\gamma_{x}^{h}(t)=\gamma_{x}(t) \cdot h_{t}= \begin{cases}t N+x_{j} e_{j}+h_{t}+Q\left(t N, h_{t}\right) & \text { if } 0 \leq t \leq t_{1}, \\ t_{1} N+\left(t-t_{1}+x_{j}\right) e_{j}+h_{t}+Q\left(t_{1} N, h_{t}\right) & \text { if } t \geq t_{1}\end{cases}
$$

forms a curvilinear cone with core $\gamma_{x}$. By the triangle inequality, if $\lambda$ is small enough, then for any $h \in \mathcal{U}(\lambda)$, the curve $t \mapsto \gamma_{x}^{h}(t)$ is a John curve starting from $x$. From now on we assume that $\lambda$ has been fixed small enough in order to ensure this property.

10. Two cases must be distinguished:

(A) $d(x, y) \leq \eta|\nu|$

(B) $d(x, y)>\eta|\nu|$.

The parameter $\eta \in(0,1)$ will be fixed later. Note that if 0 is a characteristic point, then Case $\mathbf{A}$ is empty. In Case $\mathbf{A}$ the curves $\gamma_{x}^{h}$ and $\gamma_{y}$ will meet in their first (rectifiable) piece. In Case B they will meet in their second (non-rectifiable) piece.

11. Study of Case A. We claim that there exist $\eta>0$ and $M>1$ such that for all $x$ and $y$ there exists $h \in \mathcal{U}(\lambda)$ such that $\gamma_{y}(M d(x, y))=\gamma_{x}^{h}(M d(x, y))$. A correct choice of $\eta \in(0,1)$ and $M>1$ will show that the two curves meet in their first tract (see condition (3.27) ).

Without loss of generality, we can assume $|\nu| \leq|\bar{\nu}|$ (otherwise the roles of $x$ and $y$ should be interchanged). If $t \leq t_{1}=\sigma|\nu|$, then $t \leq \bar{t}_{1}=\sigma|\bar{\nu}|$ and $\gamma_{y}(t)=\gamma_{x}^{h}(t)$ reads

$$
\hat{y}_{j}+t \bar{N}+y_{j} e_{j}+Q(y, t \bar{N})=t N+x_{j} e_{j}+h_{t}+Q\left(t N, h_{t}\right) .
$$

We have to show that the solution $h=\left(h^{\prime}, h^{\prime \prime}\right)$ of this equation belongs to $\mathcal{U}(\lambda)$ if $t=M d(x, y)$ and $M$ is large enough.

As $t \leq t_{1}$, then $\delta(t)=t$ and $h_{t}=\left(t h^{\prime}, t^{2} h^{\prime \prime}\right)$. Projecting (3.19) along the first $m$ components we get the equation $y^{\prime}+t \bar{N}=t N+h_{t}^{\prime}$, that is,

$$
t h^{\prime}=y^{\prime}+t(\bar{N}-N) \text {. }
$$

Replacing $t=M d(x, y)$ we find that the solution $h^{\prime}$ satisfies

$$
\left|h^{\prime}\right| \leq \frac{\left|y^{\prime}\right|}{M d(x, y)}+|N-\bar{N}|
$$

First, notice that $d(x, y) \simeq\left\|\left(-x_{j} e_{j}\right) \cdot\left(y_{j} e_{j}+\hat{y}_{j}\right)\right\| \geq\left|y^{\prime}\right|$, which gives $\frac{\left|y^{\prime}\right|}{\operatorname{Md}(x, y)} \lesssim$ $\frac{1}{M}$. Moreover, using the inequality $\left|\frac{v}{|v|}-\frac{w}{|w|}\right| \leq 2 \frac{|v-w|}{|v|}$ if $v, w \in \mathbb{R}^{n} \backslash\{0\}$, and the 
explicit form (3.15) and (3.16) of $\nu$ and $\bar{\nu}$, we get

$$
\begin{aligned}
|N-\bar{N}| & \leq 2 \frac{|\nu-\bar{\nu}|}{|\nu|} \\
& \leq \frac{2}{|\nu|} \sum_{i=1}^{m}\left|\partial_{i} \varphi(0)-\partial_{i} \varphi\left(\hat{y}_{j}\right)+q_{i j}(y)-\sum_{k>m, k \neq j} q_{i k}(y) \partial_{k} \varphi\left(\hat{y}_{j}\right)\right| \\
& \lesssim \frac{1}{|\nu|}\left(\left|\hat{y}_{j}\right|+\left|y^{\prime}\right|\right) \lesssim \frac{d(x, y)}{|\nu|} .
\end{aligned}
$$

The last string of estimates follows from the boundedness of $\partial_{i} \varphi, i=m+1, \ldots, n$ and $i \neq j$, from the Lipschitz continuity of $\partial_{i} \varphi, i=1, \ldots, m$, and from the inequality

$$
d(x, y) \simeq\left\|\left(-x_{j} e_{j}\right) \cdot\left(\hat{y}_{j}+y_{j} e_{j}\right)\right\|=\left\|\hat{y}_{j}+\left(y_{j}-x_{j}\right) e_{j}\right\| \geq\left\|\hat{y}_{j}\right\| \gtrsim\left|\hat{y}_{j}\right|,
$$

which holds because $y$ lies in a bounded set.

Putting (3.22) into (3.21) and using Case A we get

$$
\left|h^{\prime}\right| \lesssim \frac{1}{M}+\frac{d(x, y)}{|\nu|} \leq \frac{1}{M}+\eta
$$

This shows that $\left|h^{\prime}\right| \leq \lambda$ as soon as $M$ is large enough and $\eta$ is small enough.

We project now (3.19) along the components of the second slice obtaining

$$
\hat{y}_{j}^{\prime \prime}+y_{j} e_{j}+t Q(y, \bar{N})=x_{j} e_{j}+h_{t}^{\prime \prime}+t Q\left(N, h_{t}^{\prime}\right) .
$$

Here $h_{t}^{\prime \prime}=t^{2} h^{\prime \prime}$ and $h_{t}^{\prime}=t h^{\prime}$ where $h^{\prime}$ is the vector determined in (3.20) and satisfies the estimate (3.24). The last equation has a unique solution $h^{\prime \prime}$ which satisfies

$$
\left|h^{\prime \prime}\right| \leq \frac{\left|\hat{y}_{j}^{\prime \prime}\right|+\left|y_{j}-x_{j}\right|}{t^{2}}+\frac{1}{t}|Q(y, \bar{N})|+\left|Q\left(N, h^{\prime}\right)\right| .
$$

Here we have to replace $t=M d(x, y)$ but first we notice that, as in (3.23),

$$
d(x, y) \simeq\left\|\hat{y}_{j}+\left(y_{j}-x_{j}\right) e_{j}\right\| \geq\left|\hat{y}_{j}^{\prime \prime}\right|^{1 / 2}+\left|y_{j}-x_{j}\right|^{1 / 2} .
$$

Moreover, $|Q(y, \bar{N})| \lesssim\left|y^{\prime}\right| \lesssim d(x, y)$ and, by (3.24), $\left|Q\left(N, h^{\prime}\right)\right| \lesssim\left|h^{\prime}\right| \lesssim \frac{1}{M}+\eta$. Putting all these estimates together we find

$$
\left|h^{\prime \prime}\right| \lesssim \frac{1}{M^{2}}+\frac{1}{M}+\eta
$$

Thus $\left|h^{\prime \prime}\right| \leq \lambda$ as soon as $M$ is large enough and $\eta$ is small enough.

Our claim will be proved if we show that the choice of $M$ and $\eta$ is compatible with the condition $M d(x, y) \leq t_{1}=\sigma|\nu|$. As we are in Case A, then $d(x, y) \leq \eta|\nu|$ and we find the stronger condition

$$
M \eta \leq \sigma,
$$

which can be satisfied, taking if necessary a smaller $\eta$.

12. In view of Proposition 2.6 we have to estimate the diameter of the curves $\gamma_{x}^{h}$ and $\gamma_{y}$. First, by (3.5) we have $\operatorname{diam}\left(\gamma_{x}\right) \simeq M d(x, y)$. Moreover, if $0 \leq s, t \leq$ $M d(x, y)$ and $\|h\| \leq 1$, then

$$
\begin{aligned}
d\left(\gamma_{x}^{h}(s), \gamma_{x}^{h}(t)\right) & \leq d\left(\gamma_{x}^{h}(s), \gamma_{x}(s)\right)+d\left(\gamma_{x}(s), \gamma_{x}(t)\right)+d\left(\gamma_{x}(t), \gamma_{x}^{h}(t)\right) \\
& \lesssim\left\|h_{s}\right\|+\operatorname{diam}\left(\gamma_{x}\right)+\left\|h_{t}\right\| \lesssim d(x, y)
\end{aligned}
$$

and thus $\operatorname{diam}\left(\gamma_{x}^{h}\right) \lesssim d(x, y)$. 
13. Study of Case B. In this case the points $x$ and $y$ satisfy $d(x, y) \geq \eta|\nu|$ where $\eta>0$ is from now on a fixed constant. Recall that $t_{1}=\sigma|\nu|$ and $\bar{t}_{1}=\sigma|\bar{\nu}|$, and for $R>0$ let

$$
t_{x}=t_{1}+R^{2} d(x, y)^{2} \quad \text { and } \quad t_{y}=\bar{t}_{1}+R^{2} d(x, y)^{2} .
$$

As above let $\mathcal{U}(\lambda)=\left\{h \in \mathbb{R}^{n}:\|h\| \leq \lambda\right\}$ and $h_{t}=\left(\delta(t) h^{\prime}, \delta(t)^{2} h^{\prime \prime}\right)$ where now $\delta(t)=t_{1}+\left(t-t_{1}\right)^{1 / 2} \simeq d(\gamma(t), x)$ for $t \geq t_{1}$.

14. We claim that there exists $R>0$ such that for all $x, y$ there exists $h \in \mathcal{U}(\lambda)$ ( $\lambda$ is the parameter fixed at the end of 9) such that $\gamma_{y}\left(t_{y}\right)=\gamma_{x}^{h}\left(t_{x}\right)$ (the times $t_{x}$ and $t_{y}$ depend on $R$ ).

This equation gives

$$
\begin{aligned}
\hat{y}_{j}+\bar{t}_{1} \bar{N}+\left(t_{y}-\bar{t}_{1}+y_{j}\right) e_{j}+Q\left(y, \bar{t}_{1} \bar{N}\right)=t_{1} N & +\left(t_{x}-t_{1}+x_{j}\right) e_{j} \\
& +h_{t_{x}}+Q\left(t_{1} N, h_{t_{x}}\right) .
\end{aligned}
$$

Replacing $\bar{t}_{1}=\sigma|\bar{\nu}|, t_{1}=\sigma|\nu|, t_{y}-\bar{t}_{1}=R^{2} d(x, y)^{2}$ and $t_{x}-t_{1}=R^{2} d(x, y)^{2}$ we find

$$
\begin{aligned}
\hat{y}_{j} & +\sigma \bar{\nu}+\left(R^{2} d(x, y)^{2}+y_{j}\right) e_{j}+\sigma|\bar{\nu}| Q(y, \bar{N}) \\
& =\sigma \nu+\left(R^{2} d(x, y)^{2}+x_{j}\right) e_{j}+h_{t_{x}}+\sigma|\nu| Q\left(N, h_{t_{x}}\right) .
\end{aligned}
$$

Projecting this equation along the coordinates of the first slice we get

$$
y^{\prime}+\sigma \bar{\nu}=\sigma \nu+h_{t_{x}}^{\prime},
$$

and the solution $h_{t_{x}}^{\prime}$ satisfies

$$
\left|h_{t_{x}}^{\prime}\right| \leq\left|y^{\prime}\right|+\sigma|\nu|+\sigma|\bar{\nu}|
$$

We use $\left|y^{\prime}\right| \leq d(x, y)$ and $\sigma|\nu| \leq \sigma d(x, y) / \eta$ (this is Case B). By (3.16)

$$
\begin{aligned}
\left|\bar{\nu}_{i}\right| & \leq\left|\partial_{i} \varphi\left(\hat{y}_{j}\right)\right|+\left|q_{i j}(y)\right|+\sum_{k>m, k \neq j}\left|q_{i k}(y) \partial_{k} \varphi\left(\hat{y}_{j}\right)\right| \\
& \lesssim\left|\partial_{i} \varphi(0)\right|+\left|\partial_{i} \varphi(0)-\partial_{i} \varphi\left(\hat{y}_{j}\right)\right|+\left|y^{\prime}\right| \\
& \lesssim|\nu|+d(x, y) \lesssim \frac{d(x, y)}{\eta}
\end{aligned}
$$

because $\partial_{k} \varphi, k>m$ and $k \neq j$, are bounded functions, $\partial_{i} \varphi, i=1, \ldots, m$, are Lipschitz continuous, and $\left|\partial_{i} \varphi\left(\hat{y}_{j}\right)-\partial_{i} \varphi(0)\right| \lesssim\left|\hat{y}_{j}\right| \lesssim d(x, y)$ by (3.23). Ultimately, we obtain for some large but absolute constant $C_{0}$,

$$
\left|h_{t_{x}}^{\prime}\right| \leq C_{0} \frac{d(x, y)}{\eta}=C_{0} d(x, y)
$$

(the parameter $\eta$ has been fixed in 11 and can be considered from now on an absolute constant).

Projecting (3.28) along the coordinates of the second slice we have

$$
\hat{y}_{j}^{\prime \prime}+y_{j} e_{j}+\sigma|\bar{\nu}| Q(y, \bar{N})=x_{j} e_{j}+h_{t_{x}}^{\prime \prime}+\sigma|\nu| Q\left(N, h_{t_{x}}\right) .
$$

Thus

$$
h_{t_{x}}^{\prime \prime}=\hat{y}_{j}^{\prime \prime}+\left(y_{j}-x_{j}\right) e_{j}+\sigma|\bar{\nu}| Q(y, \bar{N})-\sigma|\nu| Q\left(N, h_{t_{x}}^{\prime}\right),
$$

where $h_{t_{x}}^{\prime}$ satisfies (3.31). Notice that by (3.25) $\left|\hat{y}_{j}^{\prime \prime}\right|+\left|y_{j}-x_{j}\right| \lesssim d(x, y)^{2}$ and moreover, taking into account (3.31) and Case B,

$$
\sigma|\nu|\left|Q\left(N, h_{t_{x}}^{\prime}\right)\right| \lesssim|\nu|\left|h_{t_{x}}^{\prime}\right| \lesssim d(x, y)^{2} .
$$


By (3.30) $\sigma|\bar{\nu}|\left|Q\left(y^{\prime}, \bar{N}\right)\right| \lesssim|\bar{\nu}|\left|y^{\prime}\right| \lesssim d(x, y)^{2}$, and hence $\left|h_{t_{x}}^{\prime \prime}\right| \lesssim d(x, y)^{2}$. Finally,

$$
\|h\|=\frac{\left\|h_{t_{x}}\right\|}{\delta\left(t_{x}\right)}=\frac{\left|h_{t_{x}}^{\prime}\right|+\left|h_{t_{x}}^{\prime \prime}\right|^{1 / 2}}{\delta\left(t_{x}\right)} \lesssim \frac{d(x, y)}{\delta\left(t_{x}\right)}=\frac{d(x, y)}{t_{1}+\left(t_{x}-t_{1}\right)^{1 / 2}} \leq \frac{1}{R},
$$

and $\|h\| \leq \lambda$ as soon as $R \geq C_{0} / \lambda$ where $C_{0}$ is a large but absolute constant.

15. The estimates for $\operatorname{diam}\left(\gamma_{x}^{h}\right)$ and $\operatorname{diam}\left(\gamma_{y}\right)$ can be obtained as in 12. Our claims are proved and the proof of the theorem in the characteristic case is ended.

16. Case 2. We now study the non-characteristic case. Assume without loss of generality that $\Omega=\left\{y \in \mathbb{R}^{n}: y_{j}>\varphi\left(\hat{y}_{j}\right)\right\}$ where $j \in\{1, \ldots, m\}$ and $\varphi \in C^{1}\left(\mathbb{R}^{n-1}\right)$ is a function such that $\varphi(0)=0,|\nabla \varphi| \leq k$ and $\partial_{i} \varphi, i=1, \ldots, m$ and $i \neq j$, are $k$-Lipschitz continuous functions. Let $\nu_{i}=-\partial_{i} \varphi(0)$ if $i=1, \ldots, m$ with $i \neq j$, and $\nu_{j}=1$. Finally, let $\nu=\left(\nu_{1}, \ldots, \nu_{m}, 0, \ldots, 0\right)$.

17. First step. We construct John curves starting from near the boundary. The function $\psi$ defined by

$$
\psi\left(\hat{y}_{j}\right)=\varphi\left(\hat{y}_{j}\right)+\sum_{i=1, \ldots, m, i \neq j} \nu_{i} y_{i}
$$

satisfies

$$
\begin{aligned}
\left|\psi\left(\hat{y}_{j}\right)\right| & =\left|\varphi\left(\hat{y}_{j}\right)-\sum_{i=1, \ldots, m, i \neq j} \partial_{i} \varphi(0) y_{i}\right| \\
& =\left|\sum_{i>m} \partial_{i} \varphi(0) y_{i}+O\left(\left|\hat{y}_{j}\right|^{2}\right)\right| \lesssim\left\|\hat{y}_{j}\right\|^{2},
\end{aligned}
$$

because $\partial_{i} \varphi, i>m$, are bounded and $\partial_{i} \varphi, i=1, \ldots, m$ and $i \neq j$, are Lipschitz continuous.

Fix a point $x \in \Omega$ of the form $x=x_{j} e_{j}$ with $x_{j}>0$. For $t \geq 0$ define the curve starting from $x$,

$$
\gamma_{x}(t)=x \cdot t \nu=x \cdot\left(t e_{j}+t \sum_{i=1, \ldots m, i \neq j} \nu_{i} e_{i}\right) .
$$

Note first that $d(\gamma(t), x) \simeq\|t \nu\|=t|\nu| \simeq t$.

18. We claim that there exist $t_{0}>0$ and $\lambda \in(0,1)$ such that for all $t \leq t_{0}$,

$$
\operatorname{dist}(\gamma(t) ; \partial \Omega) \geq \lambda t
$$

The John condition (3.35) is equivalent to $\operatorname{Box}(\gamma(t), \lambda t) \cap \partial \Omega=\varnothing$.

Points in $\operatorname{Box}(\gamma(t), \lambda t)$ are of the form

$$
\begin{aligned}
x \cdot t \nu \cdot h & =x_{j} e_{j} \cdot\left(t \nu+h+t Q\left(\nu, h^{\prime}\right)\right) \\
& =x_{j} e_{j}+t \nu+h+t Q\left(\nu, h^{\prime}\right)+Q\left(x_{j} e_{j}, t \nu+h^{\prime}\right)=: z,
\end{aligned}
$$

where $h \in \mathbb{R}^{n}$ and $\|h\| \leq \lambda t$. We have to check that $(z$ is defined in the last equation)

$$
x_{j}+t+h_{j}>\varphi\left(\hat{z}_{j}\right)=-\sum_{i \leq m, i \neq j} \nu_{i}\left(t \nu_{i}+h_{i}\right)+\psi\left(\hat{z}_{j}\right),
$$

by (3.32). Since $\left|h_{k}\right|<\lambda t, k=1, \ldots, m$, if $\lambda>0$ is small enough, the last inequality is ensured by

$$
x_{j}+(1-\lambda) t+t \sum_{i \leq m, i \neq j}\left(\nu_{i}^{2}-\lambda\left|\nu_{i}\right|\right) \geq\left|\psi\left(\hat{z}_{j}\right)\right|,
$$


which is implied by

$$
\varepsilon_{0}\left(x_{j}+t\right) \geq\left|\psi\left(\hat{z}_{j}\right)\right| .
$$

The right-hand side of (3.36) can be estimated by (3.33)

$$
\begin{aligned}
\left|\psi\left(\hat{z}_{j}\right)\right| & \lesssim\left\|\hat{z}_{j}\right\|^{2}=\left\|t \hat{\nu}_{j}+\hat{h}_{j}+t Q\left(\nu, h^{\prime}\right)+Q\left(x_{j} e_{j}, t \nu+h^{\prime}\right)\right\|^{2} \\
& \lesssim t^{2}+\lambda t^{2}+\left\|t Q\left(\nu, h^{\prime}\right)\right\|^{2}+\left\|Q\left(x_{j} e_{j}, t \nu+h^{\prime}\right)\right\|^{2} \\
& \lesssim t^{2}+(t|\nu| \lambda t)+\left(x_{j}\left|t \nu+h^{\prime}\right|\right) \lesssim t^{2}+x_{j} t,
\end{aligned}
$$

where we used $|\nu| \lesssim 1$. Then (3.36) is ensured by

$$
\epsilon_{0}\left(x_{j}+t\right) \geq t^{2}+x_{j} t
$$

where $\epsilon_{0}>0$ is a small but absolute constant. This inequality is trivially satisfied as soon as $t \leq \epsilon_{0}$.

19. Second step. We prove the uniform condition. Given two points $x, y \in \Omega$ we have to connect them by curves $\gamma_{x}$ and $\gamma_{y}$ satisfying the hypotheses of Proposition 2.6. Assume that $x=x_{j} e_{j}$ with $x_{j}>0$ and let $y=\hat{y}_{j}+y_{j} e_{j}$ with $y_{j}>\varphi\left(\hat{y}_{j}\right)$.

We first notice that if $d(x, y)<\operatorname{dist}(x ; \partial \Omega)$, then $x$ and $y$ can be connected simply by a geodesic. Therefore, without loss of generality we can assume that

$$
d(x, y) \geq \operatorname{dist}(x ; \partial \Omega) .
$$

20. We claim that there exists a constant $C_{0}>0$ such that

$$
x_{j} \leq C_{0} d(x, y)
$$

for all $x=x_{j} e_{j}, y \in \Omega$ satisfying (3.37) and lying in a bounded set (say the unit Euclidean ball centered at the origin). Indeed, if $\xi=\hat{\xi}_{j}+\varphi\left(\hat{\xi}_{j}\right) e_{j} \in \partial \Omega$, then

$$
\begin{aligned}
d(x, \xi) & \simeq\left\|\left(-x_{j} e_{j}\right) \cdot\left(\hat{\xi}_{j}+\varphi\left(\hat{\xi}_{j}\right) e_{j}\right)\right\| \\
& \simeq\left|\varphi\left(\hat{\xi}_{j}\right)-x_{j}\right|+\left|\hat{\xi}_{j}^{\prime}\right|+\left|\xi^{\prime \prime}+Q\left(-x_{j} e_{j}, \hat{\xi}_{j}+\varphi\left(\hat{\xi}_{j}\right) e_{j}\right)\right|^{1 / 2} \\
& =\left|\varphi\left(\hat{\xi}_{j}\right)-x_{j}\right|+\left|\hat{\xi}_{j}^{\prime}\right|+\left|\xi^{\prime \prime}+Q\left(-x_{j} e_{j}, \hat{\xi}_{j}^{\prime}\right)\right|^{1 / 2} .
\end{aligned}
$$

We used here the bilinearity of $Q$ and the property $0=\left(-e_{j}\right) \cdot e_{j}=-Q\left(e_{j}, e_{j}\right)$. In order to prove (3.38) it will be enough to show that

$$
x_{j} \leq C_{0}\left(\left|\varphi\left(\hat{\xi}_{j}\right)-x_{j}\right|+\left|\hat{\xi}_{j}^{\prime}\right|+\left|\xi^{\prime \prime}+Q\left(-x_{j} e_{j}, \hat{\xi}_{j}^{\prime}\right)\right|^{1 / 2}\right) .
$$

By the Lipschitz continuity of $\varphi$ we find

$$
\begin{aligned}
x_{j} & \leq\left|x_{j}-\varphi\left(\hat{\xi}_{j}\right)\right|+\left|\varphi\left(\hat{\xi}_{j}\right)\right| \lesssim\left|x_{j}-\varphi\left(\hat{\xi}_{j}\right)\right|+\left|\hat{\xi}_{j}\right| \\
& \simeq\left|x_{j}-\varphi\left(\hat{\xi}_{j}\right)\right|+\left|\hat{\xi}_{j}^{\prime}\right|+\left|\xi^{\prime \prime}\right| \\
& \lesssim\left|x_{j}-\varphi\left(\hat{\xi}_{j}\right)\right|+\left|\hat{\xi}_{j}^{\prime}\right|+\left|\xi^{\prime \prime}+Q\left(-x_{j} e_{j}, \hat{\xi}_{j}^{\prime}\right)\right|+\left|Q\left(-x_{j} e_{j}, \hat{\xi}_{j}^{\prime}\right)\right| \\
& \lesssim\left|x_{j}-\varphi\left(\hat{\xi}_{j}\right)\right|+\left|\hat{\xi}_{j}^{\prime}\right|+\left|\xi^{\prime \prime}+Q\left(-x_{j} e_{j}, \hat{\xi}_{j}^{\prime}\right)\right|^{1 / 2}+x_{j}\left|\hat{\xi}_{j}^{\prime}\right| \\
& \lesssim\left|x_{j}-\varphi\left(\hat{\xi}_{j}\right)\right|+\left|\hat{\xi}_{j}^{\prime}\right|+\left|\xi^{\prime \prime}+Q\left(-x_{j} e_{j}, \hat{\xi}_{j}^{\prime}\right)\right|^{1 / 2} .
\end{aligned}
$$

We used here the fact that all the involved vectors lie in a bounded set. Our claim (3.38) is proved.

21. Our next step is to compute the "canonical" John curve starting from a generic point $y \in \Omega$. The point $y$ and the boundary of $\Omega$ will be translated by a suitable vector $\eta \in \mathbb{R}^{n}$ in such a way that $\eta \cdot y$ lies in the half axis $\left\{\alpha e_{j}: \alpha>0\right\}$. Using the equation of the translated surface the correct vector of parameters $\bar{\nu}$ can 
be computed and the curve starting from $y$ will be defined as $\gamma_{y}(t)=\eta^{-1} \cdot(\eta \cdot y)$. $(t \bar{\nu})=y \cdot(t \bar{\nu})$ for $t \geq 0$.

22. We claim that there exist $\varrho>0$ and $C_{0}>1$ such that for all $y \in \Omega \cap\{|y| \leq \varrho\}$ there exists $\eta \in \mathbb{R}^{n}$ such that:

(i) $\eta \cdot \partial \Omega$ contains the origin;

(ii) $\eta \cdot y$ belongs to $\left\{\alpha e_{j}: \alpha>0\right\}$;

(iii) $|\eta| \leq C_{0}\left|\hat{y}_{j}\right|$.

We look for $\eta=\left(\eta^{\prime}, \eta^{\prime \prime}\right)$. If $\eta^{\prime \prime}$ is given, and we define $\eta^{\prime}$ by the equation

$$
\eta^{\prime}=-\hat{y}_{j}^{\prime}-\varphi\left(\hat{y}_{j}^{\prime}-\eta^{\prime \prime}\right) e_{j}
$$

then (i) is satisfied. Indeed, $z:=\hat{y}_{j}^{\prime}-\eta^{\prime \prime}+\varphi\left(\hat{y}_{j}^{\prime}-\eta^{\prime \prime}\right) e_{j} \in \partial \Omega$ and

$$
\eta \cdot z=\eta^{\prime}+\eta^{\prime \prime}+\hat{y}_{j}^{\prime}-\eta^{\prime \prime}+\varphi\left(\hat{y}_{j}^{\prime}-\eta^{\prime \prime}\right) e_{j}+Q\left(\eta^{\prime}, \hat{y}_{j}^{\prime}+\varphi\left(\hat{y}_{j}^{\prime}-\eta^{\prime \prime}\right) e_{j}\right)=0,
$$

by 3.40$)$.

We shall soon prove that the implicit equation

$$
\eta^{\prime \prime}+y^{\prime \prime}+Q\left(-\hat{y}_{j}^{\prime}-\varphi\left(\hat{y}_{j}^{\prime}-\eta^{\prime \prime}\right) e_{j}, \hat{y}_{j}^{\prime}+y_{j} e_{j}\right)=0,
$$

has a solution $\eta^{\prime \prime}$. Then, the choice of $\eta^{\prime \prime}$ solution of (3.41) and of $\eta^{\prime}$ as in (3.40) ensures that the vector $\eta=\left(\eta^{\prime}, \eta^{\prime \prime}\right)$ satisfies (ii). Indeed,

$$
\begin{aligned}
\eta \cdot y & =\left(-\hat{y}_{j}^{\prime}+\eta^{\prime \prime}-\varphi\left(\hat{y}_{j}^{\prime}-\eta^{\prime \prime}\right) e_{j}\right) \cdot\left(\hat{y}_{j}^{\prime}+y^{\prime \prime}+y_{j} e_{j}\right) \\
& =y^{\prime \prime}+\eta^{\prime \prime}+\left(y_{j}-\varphi\left(\hat{y}_{j}^{\prime}-\eta^{\prime \prime}\right)\right) e_{j}+Q\left(-\hat{y}_{j}^{\prime}-\varphi\left(\hat{y}_{j}^{\prime}-\eta^{\prime \prime}\right) e_{j}, \hat{y}_{j}^{\prime}+y_{j} e_{j}\right),
\end{aligned}
$$

which belongs to the $j$ th axis if and only if (3.41) holds.

We prove the existence of a solution $\eta^{\prime \prime}$. First notice that by the bilinearity of $Q$,

$$
\begin{aligned}
Q\left(-\hat{y}_{j}^{\prime}-\varphi\left(\hat{y}_{j}^{\prime}-\eta^{\prime \prime}\right) e_{j}, y^{\prime}\right) & =Q\left(-\hat{y}_{j}^{\prime}-y_{j} e_{j}+\left(y_{j}-\varphi\left(\hat{y}_{j}^{\prime}-\eta^{\prime \prime}\right)\right) e_{j}, y^{\prime}\right) \\
& =Q\left(\left(y_{j}-\varphi\left(\hat{y}_{j}^{\prime}-\eta^{\prime \prime}\right)\right) e_{j}, y^{\prime}\right) \\
& =\left(y_{j}-\varphi\left(\hat{y}_{j}^{\prime}-\eta^{\prime \prime}\right)\right) Q\left(e_{j}, y^{\prime}\right) .
\end{aligned}
$$

The map $y^{\prime} \mapsto Q\left(e_{j}, y^{\prime}\right)$ is linear and does not depend on $y_{j}$. Thus (3.41) is equivalent to

$$
\eta^{\prime \prime}+y^{\prime \prime}+\left(y_{j}-\varphi\left(\hat{y}_{j}^{\prime}-\eta^{\prime \prime}\right)\right) Q\left(e_{j}, \hat{y}_{j}^{\prime}\right)=0 .
$$

We show that there exists $\varrho>0$ such that if $y \in \Omega$ and $|y| \leq \varrho$, then (3.42) has a solution $\eta^{\prime \prime}$ satisfying

$$
\left|\eta^{\prime \prime}\right| \leq 2\left|\hat{y}_{j}\right| .
$$

We use a fixed point argument. Letting $F\left(\eta^{\prime \prime}\right)=-y^{\prime \prime}-\left(y_{j}-\varphi\left(\hat{y}_{j}^{\prime}-\eta^{\prime \prime}\right)\right) Q\left(e_{j}, \hat{y}_{j}^{\prime}\right)$ equation (3.42) becomes $F\left(\eta^{\prime \prime}\right)=\eta^{\prime \prime}$. Let $D=\left\{\eta^{\prime \prime}:\left|\eta^{\prime \prime}\right| \leq 2\left|\hat{y}_{j}\right|\right\}$. If we show that $F(D) \subset D$, then the continuous map $F$ has a fixed point by Brouwer theorem. Indeed,

$$
\begin{aligned}
\left|F\left(\eta^{\prime \prime}\right)\right| & \leq\left|y^{\prime \prime}\right|+\left|Q\left(e_{j}, \hat{y}_{j}^{\prime}\right)\right|\left|\left(y_{j}-\varphi\left(\hat{y}_{j}^{\prime}-\eta^{\prime \prime}\right)\right)\right| \\
& \leq\left|\hat{y}_{j}\right|+C\left|\hat{y}_{j}^{\prime}\right|\left(\left|y_{j}\right|+\left|\hat{y}_{j}^{\prime}\right|+\left|\eta^{\prime \prime}\right|\right) \\
& \leq\left|\hat{y}_{j}\right|(1+4 C|y|) \leq 2\left|\hat{y}_{j}\right|,
\end{aligned}
$$

as soon as $|y| \leq \varrho=1 /(4 C)$ (here the constant $C$ depends only on the surface). 
Moreover, by (3.40) and by (3.43),

$$
\left|\eta^{\prime}\right|=\left|\hat{y}_{j}^{\prime}\right|+\left|\varphi\left(\hat{y}_{j}^{\prime}-\eta^{\prime \prime}\right)\right| \lesssim\left|\hat{y}_{j}^{\prime}\right|+\left|\eta^{\prime \prime}\right| \lesssim\left|\hat{y}_{j}\right|
$$

This proves claim (iii).

23. We compute $\bar{\nu}$ by a left translation argument. Let $\Phi(y)=y_{j}-\varphi\left(\hat{y}_{j}\right)$. The parameters $\nu$ at the point $y=0$ are given by $\nu_{i}=X_{i} \Phi(0), i=1, \ldots, m$. Then for any point $\xi=\hat{\xi}_{j}+\varphi\left(\hat{\xi}_{j}\right) e_{j}$ belonging to the surface $\{\Phi=0\}$ the parameters $\nu_{i}=\nu_{i}(\xi)$ are given by

$$
\begin{aligned}
& \nu_{i}(\xi)=\left(X_{i} \Phi\right)(\xi) \\
&= \begin{cases}\left(\partial_{j}+\sum_{k>m} q_{j k}\left(\xi^{\prime}\right) \partial_{k}\right) \Phi(\xi)=1-\sum_{k>m} q_{j k}\left(\xi^{\prime}\right) \partial_{k} \varphi\left(\hat{\xi}_{j}\right) & \text { if } i=j, \\
\left(\partial_{i}+\sum_{k>m} q_{i k}\left(\xi^{\prime}\right) \partial_{k}\right) \Phi(\xi)=-\partial_{i} \varphi(\hat{\xi})-\sum_{k>m} q_{i k}\left(\xi^{\prime}\right) \partial_{k} \varphi\left(\hat{\xi}_{j}\right) & \text { if } i \neq j .\end{cases}
\end{aligned}
$$

Let $\eta \in \mathbb{R}^{n}$ be a vector relative to $y$ as in claims (i), (ii) and (iii) of 22 . The correct value of the parameters is given by the evaluation of the previous equation at the point $-\eta$ (this is because the point $-\eta$ is taken to the origin by the left translation $\left.\tau_{\eta}\right)$. Define $\bar{\nu}_{i}=\nu_{i}(-\eta)$. Set $\bar{\nu}=\left(\bar{\nu}_{1}, \ldots, \bar{\nu}_{m}, 0, \ldots, 0\right)$. We claim that

$$
|\nu-\bar{\nu}| \lesssim\left|\hat{y}_{j}\right| \text {. }
$$

If $i \neq j$, by the Lipschitz continuity of $\varphi$ and by claim (iii),

$$
\begin{aligned}
\left|\bar{\nu}_{i}-\nu_{i}\right| & =\left|-\partial_{i} \varphi\left(-\hat{\eta}_{j}\right)-\sum_{k=m+1}^{n} q_{i k}\left(-\eta^{\prime}\right) \partial_{k} \varphi\left(-\hat{\eta}_{j}\right)+\partial_{i} \varphi(0)\right| \\
& \lesssim\left|\partial_{i} \varphi(0)-\partial_{i} \varphi\left(-\hat{\eta}_{j}\right)\right|+\sum_{k=m+1}^{n}\left|q_{i k}\left(-\eta^{\prime}\right)\right|\left|\partial_{k} \varphi\left(-\hat{\eta}_{j}\right)\right| \\
& \lesssim\left|\hat{\eta}_{j}\right|+\left|\eta^{\prime}\right| \simeq|\eta| \lesssim\left|\hat{y}_{j}\right| .
\end{aligned}
$$

The estimate of the $j$ th component of $\nu-\bar{\nu}$ is easier and we do not prove it.

24. Let $\gamma_{x}$ be the curve starting from $x=x_{j} e_{j}$ defined in (3.34) and let $\gamma_{y}$ be the curve starting from $y \in \Omega$ defined for $t \geq 0$ by

$$
\gamma_{y}(t)=y \cdot(t \bar{\nu})=\hat{y}_{j}^{\prime}+t \bar{\nu}+y_{j} e_{j}+y^{\prime \prime}+Q\left(y^{\prime}, t \bar{\nu}\right),
$$

where $\bar{\nu}$ is the vector of parameters discussed above. We now construct a cone with core $\gamma_{x}$. For $\lambda>0$ let $\mathcal{U}(\lambda)=\left\{h \in \mathbb{R}^{n}:\|h\| \leq \lambda\right\}$ and for $t \geq 0$ define $h_{t}=\left(t h^{\prime}, t^{2} h^{\prime \prime}\right)$. Note that $\left\|h_{t}\right\|=t\|h\| \simeq d\left(\gamma_{x}(t), \gamma_{x}(0)\right)\|h\|$. Finally, let

$$
\begin{aligned}
\gamma_{x}^{h}(t) & =x_{j} e_{j} \cdot(t \nu) \cdot h_{t}=x_{j} e_{j} \cdot\left(t \nu+h_{t}+Q\left(t \nu, h_{t}^{\prime}\right)\right) \\
& =x_{j} e_{j}+t \nu+h_{t}+t Q\left(\nu, h_{t}^{\prime}\right)+Q\left(x_{j} e_{j}, t \nu+h_{t}^{\prime}\right) .
\end{aligned}
$$

25. We claim that there exist $M>0$ and $\varrho>0$ such that for all $x=x_{j} e_{j} \in \Omega$ and for all $y \in \Omega$ such that $\left|\hat{y}_{j}\right| \leq \varrho$ there exists $h \in \mathcal{U}(\lambda)$ such that $\gamma_{x}^{h}(M d(x, y))=$ $\gamma_{y}(M d(x, y))$. Here $\lambda$ is a parameter small enough to ensure that for all $h \in \mathcal{U}(\lambda)$, $\gamma_{x}^{h}$ is a John curve with constant $\lambda$.

Equality $\gamma_{y}(t)=\gamma_{x}^{h}(t)$ reads

$$
\hat{y}_{j}^{\prime}+t \bar{\nu}+y_{j} e_{j}+y^{\prime \prime}+t Q\left(y^{\prime}, \bar{\nu}\right)=x_{j} e_{j}+t \nu+h_{t}+t Q\left(\nu, h_{t}^{\prime}\right)+x_{j} Q\left(e_{j}, t \nu+h_{t}^{\prime}\right) .
$$


Projecting this equation along the coordinates of the first slice we get

$$
\hat{y}_{j}^{\prime}+t \bar{\nu}+y_{j} e_{j}=x_{j} e_{j}+t \nu+t h^{\prime}
$$

and the solution $h^{\prime}$ satisfies $\left|h^{\prime}\right| \leq \frac{1}{t}\left\{\left|\hat{y}_{j}^{\prime}\right|+\left|y_{j}-x_{j}\right|+t|\nu-\bar{\nu}|\right\}$. Replacing $t=$ $M d(x, y)$ we find

$$
\left|h^{\prime}\right| \leq \frac{\left|\hat{y}_{j}^{\prime}\right|+\left|y_{j}-x_{j}\right|}{M d(x, y)}+|\nu-\bar{\nu}|
$$

By the equivalence

$$
d(x, y) \simeq\|(-x) \cdot y\| \simeq\left|y_{j}-x_{j}\right|+\left|\hat{y}_{j}^{\prime}\right|+\left|y^{\prime \prime}+Q\left(-x_{j} e_{j}, \hat{y}_{j}^{\prime}\right)\right|^{1 / 2},
$$

and by (3.44) we obtain for some absolute constant $C_{0}$,

$$
\left|h^{\prime}\right| \leq C_{0}\left(\frac{1}{M}+\varrho\right)
$$

as soon as $\left|\hat{y}_{j}\right| \leq \varrho$.

We project now (3.45) along the coordinates of the second slice obtaining

$$
y^{\prime \prime}+t Q\left(y^{\prime}, \bar{\nu}\right)=h_{t}^{\prime \prime}+t Q\left(\nu, h_{t}^{\prime}\right)+x_{j} Q\left(e_{j}, t \nu+h_{t}^{\prime}\right),
$$

where $h_{t}^{\prime}=t h^{\prime}$ and $h^{\prime}$ satisfies (3.48). We deduce that

$$
\left|h_{t}^{\prime \prime}\right| \leq\left|y^{\prime \prime}\right|+t\left|Q\left(y^{\prime}, \bar{\nu}\right)\right|+t\left|Q\left(\nu, h_{t}^{\prime}\right)\right|+x_{j}\left|Q\left(e_{j}, t \nu+h_{t}^{\prime}\right)\right| .
$$

We estimate separately each term in the right-hand side. By (3.47) and (3.38)

$$
\left|y^{\prime \prime}\right| \leq\left|y^{\prime \prime}+Q\left(-x_{j} e_{j}, \hat{y}_{j}^{\prime}\right)\right|+\left|Q\left(-x_{j} e_{j}, \hat{y}_{j}^{\prime}\right)\right| \lesssim d(x, y)^{2}+x_{j}\left|\hat{y}_{j}^{\prime}\right| \lesssim d(x, y)^{2} .
$$

Moreover, $\left|Q\left(y^{\prime}, \bar{\nu}\right)\right| \lesssim\left|y^{\prime}\right| \lesssim d(x, y)$ and by (3.48) $\left|Q\left(\nu, h_{t}^{\prime}\right)\right| \lesssim\left|h_{t}^{\prime}\right| \lesssim t\left(\frac{1}{M}+\varrho\right)$. The vectors $\nu$ and $\bar{\nu}$ are bounded. Finally, again by (3.38),$x_{j}\left|Q\left(e_{j}, t \nu+h_{t}^{\prime}\right)\right| \lesssim t d(x, y)$. Then

$$
\left|h_{t}^{\prime \prime}\right| \lesssim d(x, y)^{2}+t d(x, y)+t^{2}\left(\frac{1}{M}+\varrho\right)
$$

and replacing $t=M d(x, y)$ we finally get

$$
\left|h^{\prime \prime}\right| \lesssim \frac{1}{M^{2}}+\frac{1}{M}+\varrho
$$

which shows that $\|h\| \leq \lambda$ if $M$ is large and $\varrho$ is small enough.

\section{John DOMAins in A GROUP OF STEP 3}

In this section we study John domains in groups of step 3. In order to make explicit computations we shall study the simplest Carnot group of step 3 whose Lie algebra has the lowest dimension, which is 4 .

Consider in $\mathbb{R}^{4}$ the vector fields

$$
\begin{aligned}
& X_{1}=\partial_{1}-\frac{1}{2} x_{2} \partial_{3}-\left\{\frac{1}{12}\left(x_{1} x_{2}+\alpha x_{2}^{2}\right)+\frac{1}{2} x_{3}\right\} \partial_{4}, \\
& X_{2}=\partial_{2}+\frac{1}{2} x_{1} \partial_{3}+\left\{\frac{1}{12}\left(x_{1}^{2}+\alpha x_{1} x_{2}\right)-\frac{\alpha}{2} x_{3}\right\} \partial_{4}, \\
& X_{3}=\partial_{3}+\frac{1}{2}\left(x_{1}+\alpha x_{2}\right) \partial_{4} \\
& X_{4}=\partial_{4},
\end{aligned}
$$

where $\alpha \in \mathbb{R}$ is a real parameter. The commutation relations are

$$
\left[X_{1}, X_{2}\right]=X_{3}, \quad\left[X_{1}, X_{3}\right]=X_{4}, \quad\left[X_{2}, X_{3}\right]=\alpha X_{4},
$$


and all other commutators vanish. Thus, for any $\alpha \in \mathbb{R}$ the vector fields $X_{1}, X_{2}$ are generators of a Lie algebra of differential operators in $\mathbb{R}^{4}$ of step 3 . It can be checked that the following group law on $\mathbb{R}^{4}$ makes $X_{1}, X_{2}, X_{3}$ and $X_{4}$ left invariant:

$$
\begin{aligned}
x \cdot y=\left(x_{1}\right. & +y_{1}, x_{2}+y_{2}, x_{3}+y_{3}+\frac{1}{2}\left(x_{1} y_{2}-x_{2} y_{1}\right), \\
x_{4} & +y_{4}+\frac{1}{12}\left\{\left(y_{1}+\alpha y_{2}\right)\left(x_{2} y_{1}-x_{1} y_{2}\right)+\left(x_{1}+\alpha x_{2}\right)\left(x_{1} y_{2}-x_{2} y_{1}\right)\right\} \\
& \left.+\frac{1}{2}\left\{\left(x_{1} y_{3}-x_{3} y_{1}\right)+\alpha\left(x_{2} y_{3}-y_{2} x_{3}\right)\right\}\right) .
\end{aligned}
$$

Notice that $x^{-1}=-x$. Introduce the abbreviations

$$
\begin{aligned}
q_{1}\left(x_{1}, x_{2}, x_{3}\right) & =-\left\{\frac{1}{12}\left(x_{1} x_{2}+\alpha x_{2}^{2}\right)+\frac{1}{2} x_{3}\right\}, \\
q_{2}\left(x_{1}, x_{2}, x_{3}\right) & =\left\{\frac{1}{12}\left(x_{1}^{2}+\alpha x_{1} x_{2}\right)-\frac{\alpha}{2} x_{3}\right\}, \\
q_{3}\left(x_{1}, x_{2}\right) & =\frac{1}{2}\left(x_{1}+\alpha x_{2}\right),
\end{aligned}
$$

and

$$
\begin{aligned}
Q_{3}\left(x_{1}, x_{2}, y_{1}, y_{2}\right) & =\frac{1}{2}\left(x_{1} y_{2}-x_{2} y_{1}\right) \\
Q_{4}\left(x_{1}, x_{2}, x_{3}, y_{1}, y_{2}, y_{3}\right) & =\frac{1}{2}\left\{\left(x_{1} y_{3}-x_{3} y_{1}\right)+\alpha\left(x_{2} y_{3}-y_{2} x_{3}\right)\right\} \\
+ & \frac{1}{12}\left\{\left(y_{1}+\alpha y_{2}\right)\left(x_{2} y_{1}-x_{1} y_{2}\right)+\left(x_{1}+\alpha x_{2}\right)\left(x_{1} y_{2}-x_{2} y_{1}\right)\right\},
\end{aligned}
$$

in such a way that

$$
\begin{aligned}
x \cdot y=\left(x_{1}+y_{1}, x_{2}+y_{2}, x_{3}+y_{3}+\right. & Q_{3}\left(x_{1}, x_{2}, y_{1}, y_{2}\right), \\
& \left.x_{4}+y_{4}+Q_{4}\left(x_{1}, x_{2}, x_{3}, y_{1}, y_{2}, y_{3}\right)\right) .
\end{aligned}
$$

We denote by $d$ the Carnot-Carathéodory distance induced on $\mathbb{R}^{4}$ by $X_{1}$ and $X_{2}$ and by $B(x, r)$ the open ball centered at $x \in \mathbb{R}^{4}$ with radius $r \geq 0$. Define also the following homogeneous norm in $\mathbb{R}^{4}$,

$$
\|x\|=\left|x_{1}\right|+\left|x_{2}\right|+\left|x_{3}\right|^{1 / 2}+\left|x_{4}\right|^{1 / 3} .
$$

By a standard argument it can be proved that $d(x, y) \simeq\left\|y^{-1} \cdot x\right\|$. Define the Box

$$
\operatorname{Box}(x, r)=\left\{x \cdot y \in \mathbb{R}^{4}:\|y\| \leq r\right\} .
$$

Then it follows that there exists $c>1$ such that for all $x \in \mathbb{R}^{n}$ and $r \geq 0$,

$$
\operatorname{Box}\left(x, c^{-1} r\right) \subset B(x, r) \subset \operatorname{Box}(x, c r) .
$$

Let $S \subset \mathbb{R}^{4}$ be a 3 -dimensional surface of class $C^{1}$. If $x_{0} \in S$, there exists a neighborhood $\mathcal{U}$ of $x_{0}$ in $\mathbb{R}^{4}$ and there exists $\Phi \in C^{1}(\mathcal{U} ; \mathbb{R})$ such that $S \cap \mathcal{U}=\{x \in$ $\mathcal{U}: \Phi(x)=0\}$ and $\nabla \Phi \neq 0$ on $S \cap \mathcal{U}$. A point $x \in S \cap \mathcal{U}$ is said to be characteristic if and only if $X_{1} \Phi(x)=X_{2} \Phi(x)=0$. From a geometric point of view this means that $X_{1}$ and $X_{2}$ belong to the tangent spaces to $S$ at $x$.

Definition 4.1. A characteristic point $x \in S \cap \mathcal{U}$ is of first type if $X_{3} \Phi(x) \neq 0$. If $X_{3} \Phi(x)=0$, then it is of second type. 
If $x \in S \cap \mathcal{U}$ is a characteristic point of second type, then $X_{4} \Phi(x)=\partial_{4} \Phi(x)$ can not be 0 . Otherwise, it would be $X_{1} \Phi=\cdots=X_{4} \Phi=0$ at $x$ and this is impossible because $\nabla \Phi \neq 0$ and $X_{1}, \ldots, X_{4}$ are independent at each point.

We are interested in expressing $S$ as a graph in a neighborhood of a point $\bar{x} \in S$ after a translation that takes $\bar{x}$ to the origin. Notice that $\bar{x}$ is a characteristic point (of first, second type) of $S$ if and only if 0 is a characteristic point (of first, second type) of the translated surface $\bar{x}^{-1} \cdot S$. This is an easy consequence of the left invariance of the $X_{j}$ 's.

Now, let $S=\partial \Omega$ be the boundary of an open set of class $C^{1}$ and let $\bar{x} \in$ $\partial \Omega$. Assume that $\bar{x}$ is a characteristic point of first type. Then, for any point $\tilde{x}$ in a neighborhood of $\bar{x}$, the surface $\left(\tilde{x}^{-1} \cdot \partial \Omega\right) \cap \mathcal{V}$, where $\mathcal{V} \subset \mathbb{R}^{4}$ is a suitable neighborhood of the origin, can be parameterized by a function $x_{3}=\varphi\left(x_{1}, x_{2}, x_{4}\right)$, where $\varphi \in C^{1}(D)$ and $D$ is a neighborhood of the origin in $\mathbb{R}^{3}$.

If $\bar{x}$ is a characteristic point of second type, then the variable $x_{4}$ must be given in terms of the variables $x_{1}, x_{2}, x_{3}$. Then, for any $\tilde{x}$ near $\bar{x}$, the surface $\left(\tilde{x}^{-1} \cdot \partial \Omega\right) \cap \mathcal{V}$, can be parameterized by a function $x_{4}=\varphi\left(x_{1}, x_{2}, x_{3}\right)$, where $\varphi \in C^{1}(D)$ and $D$ is a neighborhood of the origin in $\mathbb{R}^{3}$.

Definition 4.2. Let $\Omega$ be a $C^{1}$ bounded connected open set. Denote by $\Sigma_{2} \subset \partial \Omega$ the characteristic set of second type. We say that $\Omega$ is admissible if

(i) there are a neighborhood $A$ in $\partial \Omega$ of $\Sigma_{2}$, a neighborhood $D$ of the origin in $\mathbb{R}^{3}$ and constants $\epsilon_{0}, k>0$ such that for any $z \in A$,

$$
z^{-1} \cdot \partial \Omega \cap(D \times]-\epsilon_{0}, \epsilon_{0}[)=\left\{x \in \mathbb{R}^{4}: x_{4}=\varphi_{z}\left(x^{\prime}\right), \quad x^{\prime}=\left(x_{1}, x_{2}, x_{3}\right) \in D\right\},
$$

where the function $\varphi_{z}$ satisfies

$$
\begin{aligned}
\mid \varphi_{z}\left(x^{\prime}\right) & -\left\langle\nabla \varphi_{z}(0), x^{\prime}\right\rangle \mid \\
& \leq k\left(\left\|x^{\prime}\right\|^{3}+\left(\left|\partial_{1} \varphi_{z}(0)\right|^{1 / 2}+\left|\partial_{2} \varphi_{z}(0)\right|^{1 / 2}+\left|\partial_{3} \varphi_{z}(0)\right|\right)\left(x_{1}^{2}+x_{2}^{2}\right)\right)
\end{aligned}
$$

(here $\left\|x^{\prime}\right\|=\left|x_{1}\right|+\left|x_{2}\right|+\left|x_{3}\right|^{1 / 2}$ and $\nabla$ denotes the Euclidean gradient);

(ii) $\partial \Omega$ is of class $C^{1,1}$ away from $\Sigma_{2}$.

The requirement (4.5) near $\Sigma_{2}$ is the key point. The natural question now is how to check it for a given surface. An answer is contained in Lemma 4.3

Write $x=\left(x_{1}, x_{2}, x_{3}, x_{4}\right)=\left(x^{\prime}, x_{4}\right) \in \mathbb{R}^{3} \times \mathbb{R}$. Thus we can split the group law (4.1) as follows:

$$
x \cdot y=\left(\left(x^{\prime} \circ y^{\prime}\right), x_{4}+y_{4}+Q_{4}\left(x^{\prime}, y^{\prime}\right)\right),
$$

where $\circ$ denotes the composition law in the Heisenberg group. Denote by $\varrho$ the control distance in the Heisenberg group.

Lemma 4.3. Let $D_{0} \subset \mathbb{R}^{3}$ be a neighborhood of the origin. Consider a $C^{1}$ function $\varphi: D_{0} \rightarrow \mathbb{R}$ and its graph $\Gamma=\left\{x_{4}=\varphi\left(x_{1}, x_{2}, x_{3}\right)=\varphi\left(x^{\prime}\right)\right\}$. Assume that $\varphi(0,0,0)=0$ and that there exists $k>0$ such that

(i) the second derivatives $X_{i} X_{j} \varphi\left(x^{\prime}\right), i, j=1,2$, exist at any point $x^{\prime} \in D$ and the functions $X_{i} X_{j} \varphi, i, j=1,2$, satisfy

$$
\left|X_{i} X_{j} \varphi\left(x^{\prime}\right)-X_{i} X_{j} \varphi\left(y^{\prime}\right)\right| \leq k \varrho\left(x^{\prime}, y^{\prime}\right), \quad x^{\prime}, y^{\prime} \in D_{0}, \quad i, j=1,2 ;
$$


(ii) writing $\Phi(x)=\Phi\left(x_{1}, x_{2}, x_{3}, x_{4}\right)=\varphi\left(x_{1}, x_{2}, x_{3}\right)-x_{4}$, for all $x=\left(x^{\prime}, \varphi\left(x^{\prime}\right)\right)$ $\in \Gamma$, the following estimate holds:

$$
\begin{gathered}
\left|X_{1}^{2} \Phi(x)\right|+\left|X_{2}^{2} \Phi(x)\right|+\left|\left(X_{1} X_{2}+X_{2} X_{1}\right) \Phi(x)\right| \\
\leq k\left(\left|X_{1} \Phi(x)\right|^{1 / 2}+\left|X_{2} \Phi(x)\right|^{1 / 2}+\left|X_{3} \Phi(x)\right|\right) .
\end{gathered}
$$

Then there is $D \subset D_{0}$ such that, for any fixed point $z^{\prime} \in D$, the surface $\left(z^{\prime}, \varphi\left(z^{\prime}\right)\right)^{-1}$. $\Gamma$ is the graph of a function $\varphi_{z}$ defined on $\left(z^{\prime}\right)^{-1} \circ D_{0}$ which satisfies (4.5) for all $x^{\prime} \in D$.

In Example 5.2 we will give an example of admissible set by means of this lemma.

Proof. Fix a neighborhood of the origin $D \subset D_{0}$ such that $D \subset\left(z^{\prime}\right)^{-1} \circ D_{0}$ for all $z^{\prime} \in D$ (i.e. $D \circ D \subset D_{0}$ ). Take a point $z^{\prime} \in D$. It is easy to check that the set $\left(z^{\prime}, \varphi\left(z^{\prime}\right)\right)^{-1} \cdot \Gamma$ is defined by

$$
\begin{aligned}
y_{4} & =\varphi\left(z^{\prime} \circ y^{\prime}\right)-\varphi\left(z^{\prime}\right)+Q_{4}\left(-z^{\prime}, z^{\prime} \circ y^{\prime}\right) \\
& =\varphi\left(z^{\prime} \circ y^{\prime}\right)-\varphi\left(z^{\prime}\right)-Q_{4}\left(z^{\prime}, y^{\prime}\right):=\varphi_{z}(y), \quad y \in\left(z^{\prime}\right)^{-1} \circ D_{0} .
\end{aligned}
$$

The equality $Q_{4}\left(-z^{\prime}, z^{\prime} \circ y^{\prime}\right)=-Q_{4}\left(z^{\prime}, y^{\prime}\right)$ is an immediate consequence of the associative property $\left(y^{\prime}, y_{4}\right)=\left(\left(z^{\prime}, z_{4}\right)^{-1} \cdot\left(z^{\prime}, z_{4}\right)\right) \cdot\left(y^{\prime}, y_{4}\right)=\left(z^{\prime}, z_{4}\right)^{-1} \cdot\left(\left(z^{\prime}, z_{4}\right)\right.$. $\left.\left(y^{\prime}, y_{4}\right)\right), y, z \in \mathbb{R}^{4}$.

By (4.2) and (4.3) the function $Q_{4}(z, y)$ can be written as

$$
\begin{aligned}
Q_{4}\left(z^{\prime}, y^{\prime}\right)= & q_{1}\left(z^{\prime}\right) y_{1}+q_{2}\left(z^{\prime}\right) y_{2}+q_{3}\left(z^{\prime}\right) y_{3} \\
& +X_{1} q_{1}\left(z^{\prime}\right) \frac{y_{1}^{2}}{2}+X_{2} q_{2}\left(z^{\prime}\right) \frac{y_{2}^{2}}{2}+\left(X_{1} q_{2}\left(z^{\prime}\right)+X_{2} q_{1}\left(z^{\prime}\right)\right) \frac{y_{1} y_{2}}{2} .
\end{aligned}
$$

We now claim that the function $y^{\prime} \mapsto \varphi\left(z^{\prime} \circ y^{\prime}\right)-\varphi\left(z^{\prime}\right)$ admits the following "homogeneous Taylor expansion":

$$
\begin{array}{r}
\varphi\left(z^{\prime} \circ y^{\prime}\right)-\varphi\left(z^{\prime}\right)=X_{1} \varphi\left(z^{\prime}\right) y_{1}+X_{2} \varphi\left(z^{\prime}\right) y_{2}+X_{3} \varphi\left(z^{\prime}\right) y_{3}+\frac{1}{2} X_{1}^{2} \varphi\left(z^{\prime}\right) y_{1}^{2} \\
+\frac{1}{2} X_{2}^{2} \varphi\left(z^{\prime}\right) y_{2}^{2}+\frac{1}{2}\left(X_{1} X_{2}+X_{2} X_{1}\right) \varphi\left(z^{\prime}\right) y_{1} y_{2}+O\left(\left\|y^{\prime}\right\|^{3}\right)
\end{array}
$$

where $O\left(\left\|y^{\prime}\right\|^{3}\right) \leq C\left\|y^{\prime}\right\|^{3}$ for all $y \in\left(z^{\prime}\right)^{-1} \circ D_{0}$ (and thus for all $\left.y^{\prime} \in D\right)$. The constant $C$ does not depend on $z^{\prime} \in D$. Now write

$$
\varphi_{z}\left(y^{\prime}\right)=\varphi_{z}\left(y_{1}, y_{2}, y_{3}\right)-\varphi_{z}\left(y_{1}, y_{2}, 0\right)+\varphi_{z}\left(y_{1}, y_{2}, 0\right)-\varphi_{z}(0,0,0)
$$

We examine the first term. By the mean value theorem

$$
\begin{aligned}
\varphi_{z}\left(y_{1}, y_{2}, y_{3}\right)-\varphi_{z}\left(y_{1}, y_{2}, 0\right) & =\varphi\left(z^{\prime} \circ\left(y_{1}, y_{2}, y_{3}\right)\right)-\varphi\left(z^{\prime} \circ\left(y_{1}, y_{2}, 0\right)\right) \\
& =\left(\partial_{3} \varphi\right)\left(z^{\prime} \circ\left(y_{1}, y_{2}, \vartheta y_{3}\right)\right) y_{3} \\
& =\left(X_{3} \varphi\right)\left(z^{\prime} \circ\left(y_{1}, y_{2}, \vartheta y_{3}\right)\right) y_{3} \\
& =X_{3} \varphi\left(z^{\prime}\right) y_{3}+\left\{X_{3} \varphi\left(z^{\prime} \circ\left(y_{1}, y_{2}, \vartheta y_{3}\right)\right)-X_{3} \varphi\left(z^{\prime}\right)\right\} y_{3},
\end{aligned}
$$

where $\vartheta \in] 0,1\left[\right.$. By the Lipschitz continuity of $X_{3} \varphi$ (recall that $X_{3}=X_{1} X_{2}-$ $\left.X_{2} X_{1}\right)$ we get $\left|\left\{X_{3} \varphi\left(z^{\prime} \circ\left(y_{1}, y_{2}, \vartheta y_{3}\right)\right)-X_{3} \varphi\left(z^{\prime}\right)\right\}\right| \leq k\left\|y^{\prime}\right\|$, which multiplied by $y_{3}$ can be estimated by $\left\|y^{\prime}\right\|^{3}$.

We now look at the second piece. Let

$$
g(t)=\varphi\left(z^{\prime} \circ\left(t y_{1}, t y_{2}, 0\right)\right)=\varphi\left(\exp \left(t\left(y_{1} X_{1}+y_{2} X_{2}\right)\right)(z)\right), \quad t \in[0,1] .
$$


Condition (4.6) ensures that $g$ is of class $C^{2}$ and that

$$
\begin{aligned}
g^{\prime}(t) & =\sum_{i=1}^{2} y_{i} X_{i} \varphi\left(z^{\prime} \circ\left(t y_{1}, t y_{2}, 0\right)\right), \quad \text { and } \\
g^{\prime \prime}(t) & =\sum_{i, j=1}^{2} y_{i} y_{j} X_{i} X_{j} \varphi\left(z^{\prime} \circ\left(t y_{1}, t y_{2}, 0\right)\right) .
\end{aligned}
$$

Thus, by the Taylor formula,

$$
\begin{aligned}
\varphi\left(z^{\prime} \circ\left(t y_{1}, t y_{2}, 0\right)\right)-\varphi\left(z^{\prime}\right) & =g(1)-g(0)=g^{\prime}(0)+\frac{1}{2} g^{\prime \prime}(\vartheta) \\
& =\sum_{i=1}^{2} X_{i} \varphi\left(z^{\prime}\right) y_{i}+\frac{1}{2} \sum_{i, j=1}^{2} X_{i} X_{j} \varphi\left(z^{\prime}\right) y_{i} y_{j} \\
& +\frac{1}{2} \sum_{i, j=1}^{2}\left\{X_{i} X_{j} \varphi\left(z^{\prime} \circ\left(\vartheta y_{1}, \vartheta y_{2}, 0\right)\right)-X_{i} X_{j} \varphi\left(z^{\prime}\right)\right\} y_{i} y_{j} .
\end{aligned}
$$

In view of the Lipschiz continuity of $X_{i} X_{j} \varphi$ the last line can be easily estimated with $\left\|y^{\prime}\right\|^{3}$. Thus we have proved that (4.9) holds.

Subtracting (4.8) from (4.9) we find the Taylor expansion of $\varphi_{z}$ :

$\varphi_{z}(y)=\sum_{j=1}^{3}\left\{X_{j} \varphi\left(z^{\prime}\right)-q_{j}\left(z^{\prime}\right)\right\} y_{j}+\frac{1}{2} \sum_{i, j=1}^{2}\left\{X_{i} X_{j} \varphi\left(z^{\prime}\right)-X_{i} q_{j}\left(z^{\prime}\right)\right\} y_{i} y_{j}+O\left(\left\|y^{\prime}\right\|^{3}\right)$

Then

$$
\begin{aligned}
\partial_{i} \varphi_{z}(0) & =X_{i} \varphi\left(z^{\prime}\right)-q_{i}\left(z^{\prime}\right)=X_{i} \Phi\left(z^{\prime}, \varphi\left(z^{\prime}\right)\right), \quad i=1,2,3, \\
\partial_{i j}^{2} \varphi_{z}(0) & =\frac{1}{2}\left(X_{i} X_{j} \varphi\left(z^{\prime}\right)-X_{i} q_{j}\left(z^{\prime}\right)+X_{j} X_{i} \varphi\left(z^{\prime}\right)-X_{j} q_{i}\left(z^{\prime}\right)\right) \\
& =\frac{1}{2}\left(X_{i} X_{j}+X_{j} X_{i}\right) \Phi\left(z^{\prime}, \varphi\left(z^{\prime}\right)\right), \quad i, j=1,2 .
\end{aligned}
$$

Thus, assuming (4.7) we immediately see that (4.5) holds. This ends the proof of the lemma.

We are now ready to prove our main result.

Theorem 4.4. If $\Omega \subset \mathbb{R}^{4}$ is an admissible domain, then it is a John domain in $\left(\mathbb{R}^{4}, d\right)$.

Proof. We prove the theorem using Proposition 2.4. We shall construct "canonical" John curves starting from points near the boundary $\partial \Omega$. The proof will be split into several numbered small steps.

1. For a fixed point $\bar{x} \in \partial \Omega$, let $\mathcal{U} \subset \mathbb{R}^{4}$ be a neighborhood of $\bar{x}$ and let $\Phi \in C^{1,1}(\mathcal{U} ; \mathbb{R})$ be a local equation for $\partial \Omega \cap \mathcal{U}$. We shall distinguish three cases:

(C1) $X_{1} \Phi(\bar{x})=X_{2} \Phi(\bar{x})=0$, and $X_{3} \Phi(\bar{x}) \neq 0(\bar{x}$ is a characteristic point of first type);

(C2) $X_{1} \Phi(\bar{x})=X_{2} \Phi(\bar{x})=X_{3} \Phi(\bar{x})=0(\bar{x}$ is a characteristic point of second type);

(C3) $\left|X_{1} \Phi(\bar{x})\right|+\left|X_{2} \Phi(\bar{x})\right|>0(\bar{x}$ is a non-characteristic point of $\partial \Omega)$. 
2. Case 1. After a translation $0 \in \partial \Omega$ can be assumed to be near $\bar{x}$. Thus, in a neighborhood of $0, \partial \Omega$ can be written in the form $y_{3}=\varphi\left(y_{1}, y_{2}, y_{4}\right)$, where the function $\varphi=\varphi\left(y_{1}, y_{2}, y_{4}\right)$ is of class $C^{1,1}$ and $\varphi(0)=0$. Define

$$
\nu_{1}=-\partial_{1} \varphi(0), \nu_{2}=-\partial_{2} \varphi(0), \nu=\left(\nu_{1}, \nu_{2}\right), N_{1}=\frac{\nu_{1}}{|\nu|}, N_{2}=\frac{\nu_{2}}{|\nu|},
$$

and if $\nu=0$, simply set $N_{1}=N_{2}=0$. Moreover, let $\psi(y)=\varphi(y)+\nu_{1} y_{1}+\nu_{2} y_{2}$. Since $\varphi$ is $C^{1,1}$, we have

$$
|\psi(y)|=\left|\varphi(y)+\nu_{1} y_{1}+\nu_{2} y_{2}\right| \lesssim y_{1}^{2}+y_{2}^{2}+\left|y_{4}\right| .
$$

Consider now a point $x=\left(0,0, x_{3}, 0\right) \in \Omega$ with $0<x_{3} \leq 1$. We shall define a continuous path $\gamma:[0,1] \rightarrow \Omega$ such that $\gamma(0)=x$ and the John property $\operatorname{dist}(\gamma(t) ; \partial \Omega) \geq \lambda d(\gamma(t), x)$ holds for all $t \in[0,1]$ and for some $\lambda>0$ depending only on $\Omega$. The path will be made by two pieces.

3. First piece. Let $\sigma>0$ and define $t_{1}=\sigma|\nu|$. For $t \in\left[0, t_{1}\right]$ we define

$$
\gamma(t)=\left(0,0, x_{3}, 0\right) \cdot\left(t N_{1}, t N_{2}, 0,0\right) .
$$

Notice that $d(\gamma(t), x) \simeq t$ and that the first piece degenerates if $\nu=0$.

4. We claim that there exist $\sigma, \lambda \in(0,1)$ absolute constants such that for all $t \leq t_{1}$

$$
\operatorname{Box}(\gamma(t), \lambda t) \subset \Omega \text {. }
$$

Condition (4.11) is equivalent to the John property for $\gamma$ in this first piece. Points in $\operatorname{Box}(\gamma(t), \lambda t)$ are of the form

$$
\begin{aligned}
& \gamma(t) \cdot h=\left(0,0, x_{3}, 0\right) \cdot\left(t N_{1}, t N_{2}, 0,0\right) \cdot\left(h_{1}, h_{2}, h_{3}, h_{4}\right) \\
&=\left(0,0, x_{3}, 0\right) \cdot\left(t N_{1}+h_{1}, t N_{2}+h_{2}, h_{3}+Q_{3}\left(t N_{1}, t N_{2}, h_{1}, h_{2}\right),\right. \\
&\left.h_{4}+Q_{4}\left(t N_{1}, t N_{2}, 0, h_{1}, h_{2}, h_{3}\right)\right) \\
&=\left(t N_{1}+h_{1}, t N_{2}+h_{2}, x_{3}+h_{3}+Q_{3}\left(t N_{1}, t N_{2}, h_{1}, h_{2}\right),\right. \\
& \quad h_{4}+Q_{4}\left(t N_{1}, t N_{2}, 0, h_{1}, h_{2}, h_{3}\right) \\
&\left.\quad+Q_{4}\left(0,0, x_{3}, t N_{1}+h_{1}, t N_{2}+h_{2}, h_{3}+Q_{3}\left(t N_{1}, t N_{2}, h_{1}, h_{2}\right)\right)\right)
\end{aligned}
$$

with $h=\left(h_{1}, h_{2}, h_{3}, h_{4}\right)$ and $\|h\| \leq \lambda t$.

Now, $\gamma(t) \cdot h \in \Omega$ provided that (recall that $\varphi(z)=-\nu_{1} z_{1}-\nu_{2} z_{2}+\psi(z)$ )

$$
\begin{aligned}
x_{3}+h_{3}+ & Q_{3}\left(t N_{1}, t N_{2}, h_{1}, h_{2}\right) \geq-\nu_{1}\left(t N_{1}+h_{1}\right)-\nu_{2}\left(t N_{2}+h_{2}\right) \\
+ & \psi\left(t N_{1}+h_{1}, t N_{2}+h_{2}, h_{4}+Q_{4}\left(t N_{1}, t N_{2}, 0, h_{1}, h_{2}, h_{3}\right)\right. \\
& \left.+Q_{4}\left(0,0, x_{3}, t N_{1}+h_{1}, t N_{2}+h_{2}, h_{3}+Q_{3}\left(t N_{1}, t N_{2}, h_{1}, h_{2}\right)\right)\right) .
\end{aligned}
$$

Since $\nu_{1} N_{1}+\nu_{2} N_{2}=|\nu|$, this inequality is guaranteed by

$$
x_{3}+|\nu| t \geq\left|h_{1}\right|\left|\nu_{1}\right|+\left|h_{2}\right|\left|\nu_{2}\right|+\left|h_{3}\right|+\left|Q_{3}\left(t N_{1}, t N_{2}, h_{1}, h_{2}\right)\right|+|\psi(z)|,
$$

where $z=\left(z_{1}, z_{2}, z_{4}\right)$ denotes the argument of $\psi$ in 4.12). Note that $\left|h_{1}\right|\left|\nu_{1}\right|+$ $\left|h_{2}\right|\left|\nu_{2}\right| \leq \lambda|\nu| t$ and this term can be absorbed in the left-hand side if $\lambda$ is small. Moreover, $\left|h_{3}\right| \leq \lambda t^{2}$ and $\left|Q_{3}\left(t N_{1}, t N_{2}, h_{1}, h_{2}\right)\right| \lesssim \lambda t^{2}$. Then, in order to prove inclusion (4.11) it will be enough to show that

$$
\epsilon_{0}\left(x_{3}+|\nu| t\right) \geq \lambda t^{2}+|\psi(z)|
$$


for some $\epsilon_{0}>0$ small but absolute. We estimate $z_{1}, z_{2}$ and $z_{4}$. Clearly, $\left|z_{1}\right|=$ $\left|t N_{1}+h_{1}\right| \lesssim t$ and $\left|z_{2}\right|=\left|t N_{2}+h_{2}\right| \lesssim t$. Moreover,

$$
\begin{aligned}
\left|z_{4}\right|= & \mid h_{4}+Q_{4}\left(t N_{1}, t N_{2}, 0, h_{1}, h_{2}, h_{3}\right) \\
& \quad+Q_{4}\left(0,0, x_{3}, t N_{1}+h_{1}, t N_{2}+h_{2}, h_{3}+Q_{3}\left(t N_{1}, t N_{2}, h_{1}, h_{2}\right)\right) \mid \\
\lesssim & \lambda t^{3}+x_{3} t
\end{aligned}
$$

because $Q_{4}\left(0,0, x_{3}, \xi_{1}, \xi_{2}, \xi_{3}\right)=1 / 2\left\{\left(-x_{3} \xi_{1}\right)+\alpha\left(-\xi_{2} x_{3}\right)\right\}$.

Thus by (4.10)

$$
|\psi(z)| \lesssim z_{1}^{2}+z_{2}^{2}+\left|z_{4}\right| \lesssim t^{2}+\lambda t^{3}+x_{3} t \simeq t^{2}+x_{3} t,
$$

because $\lambda t^{3} \lesssim t^{2}$ (we assume $t \leq 1$ ).

We finally have to prove the inequality

$$
\epsilon_{0}\left(x_{3}+|\nu| t\right) \geq t^{2}+x_{3} t
$$

which holds if $t \leq \sigma|\nu|$ with $\sigma>0$ small depending only on $\Omega$.

5. Second piece. From now up to the end of Case $1 t_{1}=\sigma|\nu|$ will be fixed. For $t \geq t_{1}$ define

$$
\gamma(t)=\left(0,0, x_{3}, 0\right) \cdot\left(t_{1} N_{1}, t_{1} N_{2}, t-t_{1}, 0\right),
$$

and note that $d(\gamma(t), x) \simeq t_{1}+\left(t-t_{1}\right)^{1 / 2}$. Write $b=\left(t-t_{1}\right)^{1 / 2}$ and $\delta(t)=t_{1}+b$.

6 . We claim that there exists a positive $\lambda<1$ such that for all $t_{1} \leq t \leq 1$,

$$
\operatorname{Box}(\gamma(t), \lambda \delta(t)) \subset \Omega .
$$

Condition (4.14) is equivalent to the John property for $\gamma$ in its second piece.

Points in $\operatorname{Box}(\gamma(t), \lambda \delta(t))$ have the form

$$
\begin{aligned}
\gamma(t) \cdot h= & \left(0,0, x_{3}, 0\right) \cdot\left(t_{1} N_{1}, t_{1} N_{2}, b^{2}, 0\right) \cdot\left(h_{1}, h_{1}, h_{3}, h_{4}\right) \\
= & \left(t_{1} N_{1}, t_{1} N_{2}, x_{3}+b^{2}, Q_{4}\left(0,0, x_{3}, t_{1} N_{1}, t_{1} N_{2}, b^{2}\right)\right) \cdot\left(h_{1}, h_{2}, h_{3}, h_{4}\right) \\
= & \left(t_{1} N_{1}+h_{1}, t_{1} N_{2}+h_{2}, x_{3}+b^{2}+h_{3}+Q_{3}\left(t_{1} N_{1}, t_{1} N_{2}, h_{1}, h_{2}\right),\right. \\
& \left.Q_{4}\left(0,0, x_{3}, t_{1} N_{1}, t_{1} N_{2}, b^{2}\right)+h_{4}+Q_{4}\left(t_{1} N_{1}, t_{1} N_{2}, x_{3}+b^{2}, h_{1}, h_{2}, h_{3}\right)\right),
\end{aligned}
$$

with $h=\left(h_{1}, h_{2}, h_{3}, h_{4}\right)$ and $\|h\| \leq \lambda \delta(t)$. Now, $\gamma(t) \cdot h \in \Omega$ provided that

$$
\begin{gathered}
x_{3}+b^{2}+h_{3}+Q_{3}\left(t_{1} N_{1}, t_{1} N_{2}, h_{1}, h_{2}\right) \geq-\nu_{1}\left(t_{1} N_{1}+h_{1}\right)-\nu_{2}\left(t_{1} N_{2}+h_{2}\right) \\
+\psi\left(t_{1} N_{1}+h_{1}, t_{1} N_{2}+h_{2}, Q_{4}\left(0,0, x_{3}, t_{1} N_{1}, t_{1} N_{2}, b^{2}\right)+h_{4}\right. \\
\left.+Q_{4}\left(t_{1} N_{1}, t_{1} N_{2}, x_{3}+b^{2}, h_{1}, h_{2}, h_{3}\right)\right),
\end{gathered}
$$

which is implied by

$$
t_{1}|\nu|+x_{3}+b^{2} \geq\left|\nu_{1}\right|\left|h_{1}\right|+\left|\nu_{2}\right|\left|h_{2}\right|+\left|h_{3}\right|+\left|Q_{3}\left(t_{1} N_{1}, t_{1} N_{2}, h_{1}, h_{2}\right)\right|+|\psi(z)| \text {, }
$$

where $z=\left(z_{1}, z_{2}, z_{4}\right)$ is the argument of $\psi$ in (4.15). In order to prove (4.16) note that $\left|\nu_{1}\right|\left|h_{1}\right|+\left|\nu_{2}\right|\left|h_{2}\right| \lesssim \lambda|\nu| \delta(t) \simeq \lambda|\nu| t_{1}+\lambda|\nu| b$. The term $\lambda|\nu| t_{1}$ can be put in the left-hand side. Moreover, $\left|h_{3}\right| \leq \lambda \delta^{2}(t) \lesssim \lambda t_{1}^{2}+\lambda b^{2}$ and $\left|Q_{3}\left(t_{1} N_{1}, t_{1} N_{2}, h_{1}, h_{2}\right)\right| \leq$ $\lambda t_{1} \delta(t) \lesssim \lambda t_{1}^{2}+\lambda b^{2}$. The term $\lambda b^{2}$ can also be absorbed in the left-hand side.

Claim (4.14) will be proved if we show that for $t_{1} \leq t \leq 1$,

$$
\varepsilon_{0}\left(t_{1}|\nu|+x_{3}+b^{2}\right) \geq \lambda|\nu| b+\lambda t_{1}^{2}+|\psi(z)| .
$$


We estimate $z_{1}, z_{2}$ and $z_{4}$. First, $\left|z_{1}\right|=\left|t_{1} N_{1}+h_{1}\right| \lesssim t_{1}+\lambda \delta(t) \lesssim t_{1}+\lambda b$ and the same estimate holds for $\left|z_{2}\right|$. Moreover, writing $\delta$ instead of $\delta(t)$ and recalling (4.3) we get

$$
\begin{aligned}
\left|z_{4}\right| & =\left|Q_{4}\left(0,0, x_{3}, t_{1} N_{1}, t_{1} N_{2}, b^{2}\right)+h_{4}+Q_{4}\left(t_{1} N_{1}, t_{1} N_{2}, x_{3}+b^{2}, h_{1}, h_{2}, h_{3}\right)\right| \\
& \lesssim x_{3} t_{1}+\lambda \delta^{3}+\lambda t_{1}^{2} \delta+\lambda t_{1} \delta^{2}+\lambda\left(x_{3}+b^{2}\right) \delta \\
& \simeq x_{3} t_{1}+\lambda\left(t_{1}+b\right)^{3}+\lambda t_{1}^{2}\left(t_{1}+b\right)+\lambda t_{1}\left(t_{1}+b\right)^{2}+\lambda\left(x_{3}+b^{2}\right)\left(t_{1}+b\right) \\
& \simeq x_{3} t_{1}+\lambda t_{1}^{3}+\lambda b^{3}+\lambda x_{3} t_{1}+\lambda x_{3} b \simeq x_{3} t_{1}+\lambda t_{1}^{3}+\lambda b^{3}+\lambda x_{3} b .
\end{aligned}
$$

Then by (4.10)

$$
|\psi(z)| \lesssim\left|z_{1}\right|^{2}+\left|z_{2}\right|^{2}+\left|z_{4}\right| \leq t_{1}^{2}+\lambda b^{2}+x_{3} t_{1}+\lambda t_{1}^{3}+\lambda b^{3}+\lambda x_{3} b
$$

Thus (4.17) is implied by

$$
\begin{aligned}
\epsilon_{0}\left(t_{1}|\nu|+x_{3}+b^{2}\right) & \geq \lambda|\nu| b+t_{1}^{2}+\lambda b^{2}+x_{3} t_{1}+\lambda t_{1}^{3}+\lambda b^{3}+\lambda x_{3} b \\
& \simeq \lambda|\nu| b+t_{1}^{2}+\lambda b^{2}+x_{3} t_{1}+\lambda b^{3}+\lambda x_{3} b .
\end{aligned}
$$

Inequality (4.18) holds for $b=0$. This has been proved in (4.13) with $t=t_{1}$.

Taking a smaller constant in the left-hand side of (4.13) we can assert that (4.18) is guaranteed by

$$
\epsilon_{0}\left(t_{1}|\nu|+x_{3}+b^{2}\right) \geq \lambda|\nu| b+\lambda b^{2}+\lambda b^{3}+\lambda x_{3} b .
$$

We can estimate the right-hand side using $|\nu| \leq 1$ and $b \leq 1$ getting

$$
\lambda\left(|\nu| b+b^{2}+b^{3}+x_{3} b\right) \leq \lambda\left(|\nu|^{2}+b^{2}+x_{3}\right) .
$$

Recalling now that $t_{1}=\sigma|\nu|$ inequality (4.19) is proved for all $b \in(0,1)$ if $\lambda$ is small enough.

7. Case 2. Let $\bar{x} \in \partial \Omega$ be a characteristic point of second type. Take a point near $\bar{x}$. Translate it to the origin and write locally $\partial \Omega$ in the form $y_{4}=\varphi\left(y_{1}, y_{2}, y_{3}\right)$. Since the domain is admissible, (4.5) holds. Write

$$
\begin{aligned}
& \nu_{1}=-\partial_{1} \varphi(0), \nu_{2}=-\partial_{2} \varphi(0), \nu_{3}=-\partial_{3} \varphi(0), \nu=\left(\nu_{1}, \nu_{2}\right), \\
& N_{1}=\frac{\nu_{1}}{|\nu|}, N_{2}=\frac{\nu_{2}}{|\nu|}, N_{3}=\frac{\nu_{3}}{\left|\nu_{3}\right|}=\operatorname{sgn}\left(\nu_{3}\right) .
\end{aligned}
$$

If $\nu=0$, simply set $N_{1}=N_{2}=0$. If $\nu_{3}=0$, set $N_{3}=0$. Moreover, let $\psi(y)=$ $\varphi(y)+\nu_{1} y_{1}+\nu_{2} y_{2}+\nu_{3} y_{3}$. By (4.5) $\psi$ satisfies the following growth condition

$$
|\psi(y)| \lesssim\|y\|^{3}+\left(|\nu|^{1 / 2}+\left|\nu_{3}\right|\right)\left(y_{1}^{2}+y_{2}^{2}\right)
$$

We shall now construct the John curve starting from $x=x_{4} e_{4}, x_{4} \geq 0$. Without loss of generality (the map $z \mapsto z+\mu e_{4}, \mu \in \mathbb{R}$, is a left translation), assume that $x=0 \in \partial \Omega$. We have to define a continuous path $\gamma:[0,1] \rightarrow \Omega$ such that $\gamma(0)=0$ and $\operatorname{dist}(\gamma(t) ; \partial \Omega) \geq \lambda d(\gamma(t), 0)$ for all $t \in[0,1]$ and for some $\lambda>0$ depending only on $\Omega$. We split the path into three pieces.

8. First piece. For $\sigma>0$ let

$$
t_{1}= \begin{cases}\sigma \min \left\{|\nu|^{1 / 2},|\nu| /\left|\nu_{3}\right|\right\} & \text { if } \nu_{3} \neq 0, \\ \sigma|\nu|^{1 / 2} & \text { if } \nu_{3}=0\end{cases}
$$

and if $t \in\left[0, t_{1}\right]$, define

$$
\gamma(t)=\left(N_{1} t, N_{2} t, 0,0\right)
$$

Note that $d(\gamma(t), 0)=t$. 
9. We claim that there exist positive constants $\sigma, \lambda \in(0,1)$ such that for all $t \in\left[0, t_{1}\right]$ the following John property holds:

$$
\operatorname{Box}(\gamma(t), \lambda t) \subset \Omega \text {. }
$$

Points in $\operatorname{Box}(\gamma(t), \lambda t)$ are of the form

$$
\begin{aligned}
\gamma(t) \cdot h= & \left(N_{1} t, N_{2} t, 0,0\right) \cdot\left(h_{1}, h_{2}, h_{3}, h_{4}\right) \\
= & \left(N_{1} t+h_{1}, N_{2} t+h_{2}, h_{3}+Q_{3}\left(N_{1} t, N_{2} t, h_{1}, h_{2}\right),\right. \\
& \left.h_{4}+Q_{4}\left(N_{1} t, N_{2} t, 0, h_{1}, h_{2}, h_{3}\right)\right),
\end{aligned}
$$

with $h=\left(h_{1}, h_{2}, h_{3}, h_{4}\right)$ and $\|h\| \leq \lambda t$. Now, $\gamma(t) \cdot h \in \Omega$ if

$$
\begin{aligned}
h_{4}+Q_{4}\left(N_{1} t, N_{2} t, 0, h_{1}, h_{2}, h_{3}\right)> & -\nu_{1}\left(N_{1} t+h_{1}\right)-\nu_{2}\left(N_{2} t+h_{2}\right) \\
& -\nu_{3}\left(h_{3}+Q_{3}\left(N_{1} t, N_{2} t, h_{1}, h_{2}\right)\right) \\
+ & \psi\left(N_{1} t+h_{1}, N_{2} t+h_{2}, h_{3}+Q_{3}\left(N_{1} t, N_{2} t, h_{1}, h_{2}\right)\right),
\end{aligned}
$$

which is implied by

$$
\begin{aligned}
|\nu| t \geq\left|\nu_{1}\right|\left|h_{1}\right| & +\left|\nu_{2}\right|\left|h_{2}\right|+\left|\nu_{3}\right|\left|h_{3}\right|+\left|\nu_{3}\right|\left|Q_{3}\left(N_{1} t, N_{2} t, h_{1}, h_{2}\right)\right| \\
& +\left|\psi\left(N_{1} t+h_{1}, N_{2} t+h_{2}, h_{3}+Q_{3}\left(N_{1} t, N_{2} t, h_{1}, h_{2}\right)\right)\right| \\
& +\left|h_{4}\right|+\left|Q_{4}\left(N_{1} t, N_{2} t, 0, h_{1}, h_{2}, h_{3}\right)\right| .
\end{aligned}
$$

Recall that $\left|\nu_{1}\right|\left|h_{1}\right|+\left|\nu_{2}\right|\left|h_{2}\right| \leq \lambda|\nu| t,\left|h_{3}\right| \leq \lambda t^{2},\left|Q_{3}\left(N_{1} t, N_{2} t, h_{1}, h_{2}\right)\right| \leq \lambda t^{2}$, $\left|h_{4}\right| \leq \lambda t^{3}$ and $\left|Q_{4}\left(N_{1} t, N_{2} t, 0, h_{1}, h_{2}, h_{3}\right)\right| \leq \lambda t^{3}$. If $z=\left(z_{1}, z_{2}, z_{3}\right)$ is the argument of $\psi$ in (4.23), then we get

$$
\|z\|=\left\|\left(N_{1} t+h_{1}, N_{2} t+h_{2}, h_{3}+Q_{3}\left(N_{1} t, N_{2} t, h_{1}, h_{2}\right)\right)\right\| \lesssim t+\lambda t \simeq t,
$$

and by (4.20)

$$
|\psi(z)| \lesssim\|z\|^{3}+\left(|\nu|^{1 / 2}+\left|\nu_{3}\right|\right)\left(z_{1}^{2}+z_{2}^{2}\right) \leq t^{3}+\left(|\nu|^{1 / 2}+\left|\nu_{3}\right|\right) t^{2} .
$$

We finally get the following inequality which is stronger than (4.23)

$$
\epsilon_{0}|\nu| t \geq \lambda|\nu| t+\lambda\left|\nu_{3}\right| t^{2}+t^{3}+\left(|\nu|^{1 / 2}+\left|\nu_{3}\right|\right) t^{2}
$$

where $\epsilon_{0}<1$ is an absolute constant. Dividing by $t$ we have to show that

$$
\epsilon_{0}|\nu| \geq t^{2}+\left(|\nu|^{1 / 2}+\left|\nu_{3}\right|\right) t
$$

$(\lambda|\nu|$ has been absorbed in the left-hand side). It will be enough to determine all $t$ that solve the following two inequalities:

$$
t^{2}<\epsilon_{0}|\nu| \text { and } t\left(|\nu|^{1 / 2}+\left|\nu_{3}\right|\right)<\epsilon_{0}|\nu| .
$$

The first one gives $t \leq \epsilon_{0}|\nu|^{1 / 2}$ and the second one is consequently solved by $t\left|\nu_{3}\right| \leq$ $\epsilon_{0}|\nu|$. Claim (4.22) is proved if $t_{1}$ is as in (4.21) for a small absolute constant $\sigma>0$.

10. Second piece. From now on $t_{1}$ is fixed as in (4.21). For $\eta>0$ let

$$
t_{2}= \begin{cases}\eta \max \left\{|\nu|,\left|\nu_{3}\right|^{2}\right\} & \text { if } \nu_{3} \neq 0, \\ 0 & \text { if } \nu_{3}=0,\end{cases}
$$

and if $t \in\left[t_{1}, t_{1}+t_{2}\right]$ define

$$
\gamma(t)=\left(t_{1} N_{1}, t_{1} N_{2},\left(t-t_{1}\right) N_{3}, 0\right) .
$$

Notice that

$$
\delta(t):=t_{1}+\left(t-t_{1}\right)^{1 / 2} \simeq d(\gamma(t), 0) .
$$


In the sequel we shall sometimes write $\delta$ instead of $\delta(t)$. Moreover, let $b=\left(t-t_{1}\right)^{1 / 2}$.

11. We claim that there exist positive constants $\eta, \lambda<1$ such that for all $t \in\left[t_{1}, t_{1}+t_{2}\right]$ the following John property for $\gamma$ holds:

$$
\operatorname{Box}(\gamma(t), \lambda \delta(t)) \subset \Omega \text {. }
$$

Points in $\operatorname{Box}(\gamma(t), \lambda \delta)$ are of the form

$$
\begin{aligned}
\gamma(t) \cdot h= & \left(t_{1} N_{1}, t_{1} N_{2},\left(t-t_{1}\right) N_{3}, 0\right) \cdot\left(h_{1}, h_{2}, h_{3}, h_{4}\right) \\
= & \left(t_{1} N_{1}+h_{1}, t_{1} N_{2}+h_{2},\left(t-t_{1}\right) N_{3}+h_{3}+Q_{3}\left(t_{1} N_{1}, t_{1} N_{2}, h_{1}, h_{2}\right),\right. \\
& \left.h_{4}+Q_{4}\left(t_{1} N_{1}, t_{1} N_{2},\left(t-t_{1}\right) N_{3}, h_{1}, h_{2}, h_{3}\right)\right),
\end{aligned}
$$

with $h=\left(h_{1}, h_{2}, h_{3}, h_{4}\right)$ and $\|h\| \leq \lambda \delta$. Now, $\gamma(t) \cdot h \in \Omega$ if

$$
\begin{aligned}
h_{4}+Q_{4}\left(t_{1} N_{1},\right. & \left.t_{1} N_{2}, b^{2} N_{3}, h_{1}, h_{2}, h_{3}\right)>-\nu_{1}\left(t_{1} N_{1}+h_{1}\right)-\nu_{2}\left(t_{1} N_{2}+h_{2}\right) \\
& -\nu_{3}\left(b^{2} N_{3}+h_{3}+Q_{3}\left(t_{1} N_{1}, t_{1} N_{2}, h_{1}, h_{2}\right)\right) \\
& +\psi\left(t_{1} N_{1}+h_{1}, t_{1} N_{2}+h_{2}, b^{2} N_{3}+h_{3}+Q_{3}\left(t_{1} N_{1}, t_{1} N_{2}, h_{1}, h_{2}\right)\right),
\end{aligned}
$$

which is implied by

$$
\begin{aligned}
|\nu| t_{1}+\left|\nu_{3}\right| b^{2} & >\left|h_{4}\right|+\left|Q_{4}\left(t_{1} N_{1}, t_{1} N_{2}, b^{2} N_{3}, h_{1}, h_{2}, h_{3}\right)\right| \\
& +\left|\nu_{1}\right|\left|h_{1}\right|+\left|\nu_{2}\right|\left|h_{2}\right|+\left|\nu_{3}\right|\left|h_{3}\right|+\left|\nu_{3}\right|\left|Q_{3}\left(t_{1} N_{1}, t_{1} N_{2}, h_{1}, h_{2}\right)\right| \\
& +\left|\psi\left(t_{1} N_{1}+h_{1}, t_{1} N_{2}+h_{2}, b^{2} N_{3}+h_{3}+Q_{3}\left(t_{1} N_{1}, t_{1} N_{2}, h_{1}, h_{2}\right)\right)\right| .
\end{aligned}
$$

We estimate the right-hand side: $\left|h_{4}\right| \leq \lambda \delta^{3},\left|Q_{4}\left(t_{1} N_{1}, t_{1} N_{2}, b^{2}, h_{1}, h_{2}, h_{3}\right)\right| \leq$ $\lambda \delta^{3}+b^{2} \lambda \delta \simeq \lambda \delta^{3},\left|Q_{3}\left(t_{1} N_{1}, t_{1} N_{2}, h_{1}, h_{2}\right)\right| \leq \lambda t_{1} \delta \leq \lambda \delta^{2}$ and finally $\left|\nu_{1}\right|\left|h_{1}\right|+$ $\left|\nu_{2}\right|\left|h_{2}\right| \leq \lambda|\nu| \delta,\left|h_{3}\right| \leq \lambda \delta^{2}$.

Let $z=\left(z_{1}, z_{2}, z_{3}\right)$ be the argument of $\psi$ in (4.27). Then $\left|z_{1}\right|=\left|t_{1} N_{1}+h_{1}\right| \lesssim$ $t_{1}+\lambda \delta$ and analogously $\left|z_{2}\right| \lesssim t_{1}+\lambda \delta$. Moreover, as $b \leq \delta$ and $t_{1} \leq \delta$,

$$
\begin{aligned}
\left\|\left(z_{1}, z_{2}, z_{3}\right)\right\| & =\|\left(t_{1} N_{1}+h_{1}, t_{1} N_{2}+h_{2}, b^{2} N_{3}+h_{3}+Q_{3}\left(t_{1} N_{1}, t_{1} N_{2}, h_{1}, h_{2}\right) \|\right. \\
& \simeq t_{1}+\lambda \delta+b+\lambda \delta+\left(t_{1} \lambda \delta\right)^{1 / 2} \lesssim \delta .
\end{aligned}
$$

By 4.20) this furnishes

$$
\begin{aligned}
|\psi(z)| & \lesssim \delta^{3}+\left(|\nu|^{1 / 2}+\left|\nu_{3}\right|\right)\left(t_{1}+\lambda \delta\right)^{2} \\
& \lesssim \delta^{3}+|\nu|^{1 / 2} t_{1}^{2}+\lambda|\nu|^{1 / 2} \delta^{2}+\left|\nu_{3}\right| t_{1}^{2}+\lambda\left|\nu_{3}\right| \delta^{2}
\end{aligned}
$$

and (4.27) is guaranteed by

$|\nu| t_{1}+\left|\nu_{3}\right| b^{2} \geq \lambda \delta^{3}+\lambda|\nu| \delta+\lambda\left|\nu_{3}\right| \delta^{2}+\delta^{3}+|\nu|^{1 / 2} t_{1}^{2}+\lambda|\nu|^{1 / 2} \delta^{2}+\left|\nu_{3}\right| t_{1}^{2}+\lambda\left|\nu_{3}\right| \delta^{2}$.

Replacing $\delta=t_{1}+b$ we get

$$
\begin{aligned}
\epsilon_{0}\left(|\nu| t_{1}+\left|\nu_{3}\right| b^{2}\right) \geq\left(t_{1}+b\right)^{3} & +\lambda|\nu|\left(t_{1}+b\right)+\lambda\left|\nu_{3}\right|\left(t_{1}+b\right)^{2}+|\nu|^{1 / 2} t_{1}^{2} \\
& +\lambda|\nu|^{1 / 2}\left(t_{1}+b\right)^{2}+\left|\nu_{3}\right| t_{1}^{2},
\end{aligned}
$$

where $\epsilon_{0}$ is a small but absolute constant. Possibly changing $\epsilon_{0}$ it will be enough to show that

$$
\begin{aligned}
\epsilon_{0}\left(|\nu| t_{1}+\left|\nu_{3}\right| b^{2}\right) \geq t_{1}^{3}+b^{3} & +\lambda|\nu| t_{1}+\lambda|\nu| b+\lambda\left|\nu_{3}\right| t_{1}^{2}+\lambda\left|\nu_{3}\right| b^{2} \\
& +|\nu|^{1 / 2} t_{1}^{2}+\lambda|\nu|^{1 / 2} t_{1}^{2}+\lambda|\nu|^{1 / 2} b^{2}+\left|\nu_{3}\right| t_{1}^{2} .
\end{aligned}
$$


Now, $\lambda|\nu| t_{1}$ and $\lambda\left|\nu_{3}\right| b^{2}$ can be absorbed in the left-hand side, and $\lambda\left|\nu_{3}\right| t_{1}^{2}+\left|\nu_{3}\right| t_{1}^{2} \simeq$ $\left|\nu_{3}\right| t_{1}^{2}$. Then

$$
\epsilon_{0}\left(|\nu| t_{1}+\left|\nu_{3}\right| b^{2}\right) \geq t_{1}^{3}+b^{3}+\lambda|\nu| b+\left|\nu_{3}\right| t_{1}^{2}+|\nu|^{1 / 2} t_{1}^{2}+\lambda|\nu|^{1 / 2} b^{2} .
$$

Inequality (4.29) holds with $b=0$ by (4.24) with $t=t_{1}$. It will be enough to show that

$$
\epsilon_{0}\left(|\nu| t_{1}+\left|\nu_{3}\right| b^{2}\right) \geq b^{3}+\lambda|\nu| b+\lambda|\nu|^{1 / 2} b^{2} .
$$

12. In order to prove (4.30) the following two cases must be distinguished:

(2A) $\left|\nu_{3}\right| \leq|\nu|^{1 / 2}$

(2B) $\left|\nu_{3}\right|>|\nu|^{1 / 2}$.

13. Case 2A. In this case $t_{1}=\sigma|\nu|^{1 / 2}$ and (4.30) becomes (with a smaller $\epsilon_{0}$ )

$$
\epsilon_{0}\left(|\nu|^{3 / 2}+\left|\nu_{3}\right| b^{2}\right) \geq b^{3}+\lambda|\nu| b+\lambda|\nu|^{1 / 2} b^{2} .
$$

By the trivial estimate $\left|\nu_{3}\right| b^{2} \geq 0$ and letting $\lambda=1$ in the right-hand side we get the stronger inequality $\epsilon_{0}|\nu|^{3 / 2} \geq b^{3}+|\nu| b+|\nu|^{1 / 2} b^{2}$. Setting $b=|\nu|^{1 / 2} a$ (this can be done because in Case $2 \mathrm{~A}$ it should be $\nu \neq 0$ ) we find $\epsilon_{0} \geq a^{3}+a^{2}+a$ which holds for all $0 \leq a<a_{0}$. Then (4.30) holds for all $0 \leq b \leq a_{0}|\nu|^{1 / 2}$ and consequently our claim (4.26) holds for all $t \leq t_{1}+a_{0}^{2}|\nu|$.

14. Case 2B. Here $t_{1}=\sigma|\nu| /\left|\nu_{3}\right|$. The term $\lambda|\nu|^{1 / 2} b^{2}$ in the right-hand side of (4.30) is less than $\epsilon_{0}\left|\nu_{3}\right| b^{2}$ and can be absorbed in the left-hand side. Then we get the inequality (with a possibly smaller $\epsilon_{0}$ )

$$
\epsilon_{0}\left(\frac{|\nu|^{2}}{\left|\nu_{3}\right|}+\left|\nu_{3}\right| b^{2}\right) \geq b^{3}+\lambda|\nu| b
$$

that is,

$$
\epsilon_{0}\left(|\nu|^{2}+\left|\nu_{3}\right|^{2} b^{2}\right) \geq b^{3}\left|\nu_{3}\right|+\lambda|\nu|\left|\nu_{3}\right| b .
$$

Now, $\lambda|\nu|\left|\nu_{3}\right| b \leq \frac{\lambda}{2}|\nu|^{2}+\frac{\lambda}{2}\left|\nu_{3}\right|^{2} b^{2}$ and both these terms can be absorbed in the left-hand side if $\lambda$ is suitable. Thus it suffices to solve

$$
\epsilon_{0}\left(|\nu|^{2}+\left|\nu_{3}\right|^{2} b^{2}\right) \geq b^{3}\left|\nu_{3}\right| \text {. }
$$

Setting $|\nu|=0$ we find $b \leq \epsilon_{0}\left|\nu_{3}\right|$ which gives the correct choice $t_{2}=\epsilon_{0}^{2}\left|\nu_{3}\right|^{2}$, as declared in (4.25). Claim (4.26) is proved in Case 2B also.

15. Third piece. From now on $t_{2}$ is fixed as in 4.25. If $t \geq t_{1}+t_{2}$, define

$$
\gamma(t)=\left(t_{1} N_{1}, t_{1} N_{2}, t_{2} N_{3}, t-\left(t_{1}+t_{2}\right)\right)
$$

and notice that

$$
\delta(t):=t_{1}+t_{2}^{1 / 2}+\left(t-\left(t_{1}+t_{2}\right)\right)^{1 / 3} \simeq d(\gamma(t), 0) .
$$

As before we shall sometimes write $\delta$ instead of $\delta(t)$. Moreover, let

$$
a=\left(t-\left(t_{1}+t_{2}\right)\right)^{1 / 3} .
$$

16. We claim that there exists $\lambda<1$ such that the following John property for $\gamma$ holds for all $t_{1}+t_{2} \leq t \leq 1$ :

$$
\operatorname{Box}(\gamma(t), \lambda \delta(t)) \subset \Omega
$$


Points in $\operatorname{Box}(\gamma(t), \lambda \delta(t))$ are of the form

$$
\begin{aligned}
\gamma(t) \cdot h= & \left(t_{1} N_{1}, t_{1} N_{2}, t_{2} N_{3}, a^{3}\right) \cdot\left(h_{1}, h_{2}, h_{3}, h_{4}\right) \\
= & \left(t_{1} N_{1}+h_{1}, t_{1} N_{2}+h_{2}, t_{2} N_{3}+h_{3}+Q_{3}\left(t_{1} N_{1}, t_{1} N_{2}, h_{1}, h_{2}\right),\right. \\
& \left.a^{3}+h_{4}+Q_{4}\left(t_{1} N_{1}, t_{1} N_{2}, t_{2} N_{3}, h_{1}, h_{2}, h_{3}\right)\right),
\end{aligned}
$$

where $h=\left(h_{1}, h_{2}, h_{3}, h_{4}\right)$ and $\|h\| \leq \lambda \delta$. Now, $\gamma(t) \cdot h \in \Omega$ if

$$
\begin{aligned}
a^{3}+h_{4} & +Q_{4}\left(t_{1} N_{1}, t_{1} N_{2}, t_{2} N_{3}, h_{1}, h_{2}, h_{3}\right) \geq-\nu_{1}\left(t_{1} N_{1}+h_{1}\right)-\nu_{2}\left(t_{1} N_{2}+h_{2}\right) \\
& -\nu_{3}\left(t_{2} N_{3}+h_{3}+Q_{3}\left(t_{1} N_{1}, t_{1} N_{2}, h_{1}, h_{2}\right)\right) \\
& +\left|\psi\left(t_{1} N_{1}+h_{1}, t_{1} N_{2}+h_{2}, t_{2} N_{3}+h_{3}+Q_{3}\left(t_{1} N_{1}, t_{1} N_{2}, h_{1}, h_{2}\right)\right)\right| .
\end{aligned}
$$

As usual, we find the stronger inequality

$$
\begin{aligned}
|\nu| t_{1}+\left|\nu_{3}\right| t_{2}+a^{3} & \geq\left|h_{4}\right|+\left|Q_{4}\left(t_{1} N_{1}, t_{1} N_{2}, t_{2} N_{3}, h_{1}, h_{2}, h_{3}\right)\right| \\
& +\left|\nu_{1}\right|\left|h_{1}\right|+\left|\nu_{2}\right|\left|h_{2}\right|+\left|\nu_{3}\right|\left|h_{3}\right|+\left|\nu_{3}\right|\left|Q_{3}\left(t_{1} N_{1}, t_{1} N_{2}, h_{1}, h_{2}\right)\right| \\
& +\left|\psi\left(t_{1} N_{1}+h_{1}, t_{1} N_{2}+h_{2}, t_{2} N_{3}+h_{3}+Q_{3}\left(t_{1} N_{1}, t_{1} N_{2}, h_{1}, h_{2}\right)\right)\right| .
\end{aligned}
$$

In the right-hand side we can estimate $\left|h_{4}\right|,\left|Q_{4}\right| \lesssim \lambda \delta^{3},\left|\nu_{1}\right|\left|h_{1}\right|+\left|\nu_{2}\right|\left|h_{2}\right| \leq \lambda|\nu| \delta$, $\left|h_{3}\right| \leq \lambda \delta^{2}$ and $\left|Q_{3}\left(t_{1} N_{1}, t_{1} N_{2}, h_{1}, h_{2}\right)\right| \lesssim \lambda t_{1} \delta \lesssim \lambda \delta^{2}$.

Let $z=\left(z_{1}, z_{2}, z_{3}\right)$ be the argument of $\psi$. Then $\left|z_{1}\right|=\left|t_{1} N_{1}+h_{1}\right| \leq t_{1}+\lambda \delta$ and $\left|z_{2}\right| \leq t_{1}+\lambda \delta$. Moreover,

$$
\begin{aligned}
\|z\| & =\left\|\left(t_{1} N_{1}+h_{1}, t_{2} N_{2}+h_{2}, t_{2} N_{3}+h_{3}+Q_{3}\left(t_{1} N_{1}, t_{1} N_{2}, h_{1}, h_{2}\right)\right)\right\| \\
& \lesssim t_{1}+\lambda \delta+t_{2}^{1 / 2}+\lambda \delta+\left(t_{1} \lambda \delta\right)^{1 / 2} \simeq t_{1}+\lambda \delta+t_{2}^{1 / 2} .
\end{aligned}
$$

By (4.20)

$$
\begin{aligned}
|\psi(z)| & \leq\|z\|^{3}+\left(|\nu|^{1 / 2}+\left|\nu_{3}\right|\right)\left(z_{1}^{2}+z_{2}^{2}\right) \\
& \leq\left(t_{1}+\lambda \delta+t_{2}^{1 / 2}\right)^{3}+\left(|\nu|^{1 / 2}+\left|\nu_{3}\right|\right)\left(t_{1}+\lambda \delta\right)^{2} \\
& \simeq t_{1}^{3}+t_{2}^{3 / 2}+\lambda \delta^{3}+t_{1}^{2}|\nu|^{1 / 2}+t_{1}^{2}\left|\nu_{3}\right|+\lambda|\nu|^{1 / 2} \delta^{2}+\lambda\left|\nu_{3}\right| \delta^{2} .
\end{aligned}
$$

Ultimately, we have to show that

$$
\begin{aligned}
\epsilon_{0}\left(t_{1}|\nu|+t_{2}\left|\nu_{3}\right|+a^{3}\right) \geq \lambda \delta^{3} & +\lambda|\nu| \delta+\lambda\left|\nu_{3}\right| \delta^{2}+t_{1}^{3}+t_{2}^{3 / 2} \\
& +t_{1}^{2}|\nu|^{1 / 2}+t_{1}^{2}\left|\nu_{3}\right|+\lambda|\nu|^{1 / 2} \delta^{2} .
\end{aligned}
$$

Notice that $|\nu|^{1 / 2} \delta^{2} \leq \frac{1}{2}\left(|\nu| \delta+\delta^{3}\right)$ and thus the term $\lambda|\nu|^{1 / 2} \delta^{2}$ in the right-hand side can be deleted. Now, writing $\delta=t_{1}+t_{2}^{1 / 2}+a$ we get

$$
\begin{aligned}
\epsilon_{0}\left(t_{1}|\nu|+t_{2}\left|\nu_{3}\right|+a^{3}\right) \geq \lambda t_{1}^{3} & +\lambda t_{2}^{3 / 2}+\lambda a^{3}+\lambda|\nu| t_{1}+\lambda|\nu| t_{2}^{1 / 2}+\lambda|\nu| a+\lambda\left|\nu_{3}\right| t_{1}^{2} \\
& +\lambda\left|\nu_{3}\right| t_{2}+\lambda\left|\nu_{3}\right| a^{2}+t_{1}^{3}+t_{2}^{3 / 2}+t_{1}^{2}|\nu|^{1 / 2}+t_{1}^{2}\left|\nu_{3}\right|,
\end{aligned}
$$

and letting $\lambda|\nu| t_{1}, \lambda\left|\nu_{3}\right| t_{2}$ and $\lambda a^{3}$ be absorbed by the left-hand side we find the stronger inequality

$$
\begin{aligned}
\epsilon_{0}\left(t_{1}|\nu|+t_{2}\left|\nu_{3}\right|+a^{3}\right) \geq t_{1}^{3} & +t_{2}^{3 / 2}+\lambda|\nu| t_{2}^{1 / 2}+\lambda|\nu| a \\
& +\lambda\left|\nu_{3}\right| a^{2}+t_{1}^{2}|\nu|^{1 / 2}+t_{1}^{2}\left|\nu_{3}\right| .
\end{aligned}
$$


Such inequality holds if $a=0$ (let $b^{2}=t_{2}$ in (4.29). Thus, it will be enough to prove that for a small but absolute constant $\epsilon_{0}$,

$$
\epsilon_{0}\left(t_{1}|\nu|+t_{2}\left|\nu_{3}\right|+a^{3}\right) \geq \lambda|\nu| a+\lambda\left|\nu_{3}\right| a^{2},
$$

for all $a \geq 0$.

We distinguish Case $2 \mathrm{~A}$ and Case $2 \mathrm{~B}$.

17. Case 2A. In this case $\left|\nu_{3}\right| \leq|\nu|^{1 / 2}, t_{1}=\sigma|\nu|^{1 / 2}$ and $t_{2}=\eta|\nu|$. Using $t_{2}\left|\nu_{3}\right| \geq 0$ in the left-hand side of (4.32), replacing $t_{1}=\sigma|\nu|^{1 / 2}$ and using also $\left|\nu_{3}\right| \leq|\nu|^{1 / 2}$ in the right-hand side we get the stronger inequality

$$
\epsilon_{0}\left(|\nu|^{3 / 2}+a^{3}\right) \geq \lambda\left(|\nu| a+|\nu|^{1 / 2} a^{2}\right)
$$

which holds for all $a \geq 0$ if $\lambda$ is small enough ( 3 and $3 / 2$ are Hölder conjugate exponents).

18. Case 2B. Here $\left|\nu_{3}\right|>|\nu|^{1 / 2}, t_{1}=\sigma|\nu| /\left|\nu_{3}\right|$ and $t_{2}=\eta\left|\nu_{3}\right|^{2}$. In the left-hand side of 4.32 we use $t_{1}|\nu| \geq 0$ and put $t_{2}=\eta\left|\nu_{3}\right|^{2}$. In the right-hand side we estimate $|\nu| \leq\left|\nu_{3}\right|^{2}$. Thus we find the stronger inequality

$$
\epsilon_{0}\left(\left|\nu_{3}\right|^{3}+a^{3}\right) \geq \lambda\left|\nu_{3}\right|^{2} a+\lambda\left|\nu_{3}\right| a^{2},
$$

which holds for all $a \geq 0$ if $\lambda$ is small enough.

19. Case 3. This is the non-characteristic case and can be analyzed as in Theorem 3.2 .

\section{EXAMPLES}

Example 5.1. In the setting of the Heisenberg group we give an example of domain of class $C^{1, \alpha}$ with $\alpha \in(0,1)$ which is not a John domain. To this aim we construct a counterexample to the Sobolev-Poincaré inequality (1.1).

We consider $(x, y, t)=(z, t) \in \mathbb{R}^{2} \times \mathbb{R}=\mathbb{H}^{1}$ and the vector fields $X_{1}=\partial_{x}+y \partial_{t}$ and $X_{2}=\partial_{y}-x \partial_{t},(x, y, t)=(z, t) \in \mathbb{R}^{3}$. We shall write $X=\left(X_{1}, X_{2}\right)$. It is well known that these vector fields are associated with a homogeneous group with dimension $Q=4$.

Let $\Omega=\left\{(z, t) \in \mathbb{H}^{1}:|z|^{\alpha+1}<t<1\right\}$ where $\alpha \geq 0$ is a real parameter. The domain $\partial \Omega$ is not smooth when $|z|=t=1$ but this does not matter as we are interested in the characteristic point $0 \in \partial \Omega$. If $\alpha \geq 1$, then $\Omega$ is of class $C^{2}$ in a neighborhood of $0 \in \partial \Omega$ and it belongs to the regular class studied in Section 3 .

We consider the case $0 \leq \alpha<1$. We let $u(z, t)=t^{-\gamma}$ and look for an exponent $\gamma>0$ such that

$$
\int_{\Omega}|X u|^{p} d z d t<+\infty \text { but } \int_{\Omega}|u|^{q} d z d t=+\infty,
$$

where $1 \leq p<Q=4$, for some $q \geq 1$ which should be less than $p^{*}=4 p /(4-p)$. We have

$$
\begin{aligned}
\int_{\Omega}|X u|^{p} d z d t & \simeq \int_{0}^{1} t^{-p(\gamma+1)} \int_{|z|<t^{1 /(\alpha+1)}}|z|^{p} d z d t \\
& \simeq \int_{0}^{1} t^{-p(\gamma+1)+(2+p) /(\alpha+1)} d t<+\infty \quad \Leftrightarrow \quad \gamma<\frac{3+\alpha-p \alpha}{p(\alpha+1)} .
\end{aligned}
$$

On the other hand,

$$
\int_{\Omega}|u|^{q} d z d t \simeq \int_{0}^{1} t^{-q \gamma+4 /(\alpha+1)} d t=+\infty \quad \Leftrightarrow \quad \gamma \geq \frac{3+\alpha}{q(\alpha+1)} .
$$


An exponent $\gamma$ ensuring (5.1) can be found if the following condition holds:

$$
\frac{3+\alpha}{q(\alpha+1)}<\frac{3+\alpha-p \alpha}{p(\alpha+1)} \quad \Leftrightarrow \quad q>\frac{p(3+\alpha)}{3+\alpha-p \alpha} .
$$

If $\alpha=1$ (5.2) becomes $q>p^{*}$ which is exactly what one should expect. If $\alpha<1$, we can find $q<p^{*}$ such that (5.2) holds (the function $\alpha \mapsto(3+\alpha) p /(3+\alpha-p \alpha$ ) is increasing near $\alpha=1$ ). Thus if $\alpha<1$, the Sobolev-Poincaré inequality does not hold and $\Omega$ is not a John domain.

Example 5.2. Using Theorem 4.4 we construct an example of John domain $\Omega \subset \mathbb{R}^{4}$ with respect to the metric structure of the group of step 3 considered in Section 4 Let $g \in C^{2}(0,1) \cap C([0,1])$ be a function such that

$$
g(t)= \begin{cases}1-t^{1 / 4} & \text { if } 0 \leq t \leq 1 / 4 \\ (1-t)^{1 / 4} & \text { if } 3 / 4 \leq t \leq 1\end{cases}
$$

Such a function can be chosen with the additional property $g^{\prime}(t)<0$ for all $t \in$ $(0,1)$.

Let

$$
N\left(x_{1}, x_{2}, x_{3}\right)=\left(x_{1}^{2}+x_{2}^{2}\right)^{6}+x_{3}^{6},
$$

and define the open set

$$
\Omega=\left\{x \in \mathbb{R}^{4}:\left|x_{4}\right|<g\left(N\left(x_{1}, x_{2}, x_{3}\right)\right)\right\} .
$$

Notice that if $N\left(x_{1}, x_{2}, x_{3}\right) \geq 3 / 4$, then $\partial \Omega$ has local equation $\left(x_{1}^{2}+x_{2}^{2}\right)^{6}+x_{3}^{6}+x_{4}^{4}=$ 1. If $N\left(x_{1}, x_{2}, x_{3}\right) \leq 1 / 4$, then $\partial \Omega$ has equation $\left|x_{4}\right|+\left[\left(x_{1}^{2}+x_{2}^{2}\right)^{6}+x_{3}^{6}\right]^{1 / 4}=1$.

We first show that the points $(0,0,0, \pm 1) \in \partial \Omega$ are the unique characteristic points of second type of $\partial \Omega$. Indeed, let $\Phi\left(x_{1}, x_{2}, x_{3}, x_{4}\right)=g(N(x))-x_{4}$ and compute

$$
\begin{aligned}
& X_{1} \Phi(x)=g^{\prime}(N(x)) X_{1} N(x)-q_{1}(x), \\
& X_{2} \Phi(x)=g^{\prime}(N(x)) X_{2} N(x)-q_{2}(x), \\
& X_{3} \Phi(x)=g^{\prime}(N(x)) X_{3} N(x)-q_{3}(x),
\end{aligned}
$$

where $q_{1}, q_{2}$ and $q_{3}$ are defined in (4.2). Note that $x_{1} q_{1}(x)+x_{2} q_{2}(x)=-x_{3} q_{3}(x)$. Moreover,

$$
\begin{aligned}
& X_{1} N(x)=12 x_{1}\left(x_{1}^{2}+x_{2}^{2}\right)^{5}-3 x_{2} x_{3}^{5}, \\
& X_{2} N(x)=12 x_{2}\left(x_{1}^{2}+x_{2}^{2}\right)^{5}+3 x_{1} x_{3}^{5},
\end{aligned}
$$

and thus $x_{1} X_{1} N(x)+x_{2} X_{2} N(x)=12\left(x_{1}^{2}+x_{2}^{2}\right)^{6}$. Then

$$
x_{1} X_{1} \Phi+x_{2} X_{2} \Phi=g^{\prime}(N(x)) 12\left(x_{1}^{2}+x_{2}^{2}\right)^{6}+x_{3} q_{3}=0,
$$

if $x$ is characteristic. Moreover, if $x$ is of second type, we have

$$
x_{3} X_{3} \Phi=g^{\prime}(N(x)) 6 x_{3}^{6}-x_{3} q_{3}(x)=0 .
$$

Summing up the last two equations we finally get

$$
g^{\prime}(N(x))\left(12\left(x_{1}^{2}+x_{2}^{2}\right)^{6}+6 x_{3}^{6}\right)=0,
$$

which implies $x_{1}=x_{2}=x_{3}=0$, as $g^{\prime}(N(x)) \neq 0$. 
We now check that hypotheses of Lemma 4.3 are satisfied. We start with (i). Consider the graph $x_{4}=\varphi\left(x_{1}, x_{2}, x_{3}\right)=N(x)^{1 / 4}, x \in D_{0}=\left\{\left(x_{1}, x_{2}, x_{3}\right): N(x)<\right.$ $1\}$. We need to show that the derivatives $X_{i} X_{j} \varphi, i, j=1,2$, are Lipschitz continuous on $D_{0}$ (with respect to the distance $\varrho$ in the Heisenberg group). This is equivalent to showing that the derivatives $X_{i} X_{j} X_{k} \varphi(i, j, k=1,2)$ are bounded. This is trivially true because these functions are smooth away from the origin and homogeneous of degree zero with respect to the dilations $\left(x_{1}, x_{2}, x_{3}\right) \mapsto\left(\lambda x_{1}, \lambda x_{2}, \lambda^{2} x_{3}\right)$ (we are taking derivatives of order 3 of the function $\varphi=N^{1 / 4}$ which is homogeneous of degree 3$)$.

In order to check hypothesis (ii) of Lemma 4.3 we have to prove that, letting $\Phi(x)=N(x)^{1 / 4}-x_{4}$, there exists a constant $k>0$ such that

$$
\left|X_{1}^{2} \Phi\right|+\left|X_{2}^{2} \Phi\right|+\left|\left(X_{1} X_{2}+X_{2} X_{1}\right) \Phi\right| \leq k\left(\left|X_{1} \Phi\right|^{1 / 2}+\left|X_{2} \Phi\right|^{1 / 2}+\left|X_{3} \Phi\right|\right)
$$

for all $x \in \partial \Omega$ such that $0<N(x) \leq 1 / 4$. We note that away from the origin the function $\Phi(x)=N(x)^{1 / 4}-x_{4}$ is smooth, and moreover, it is homogeneous of degree 3 with respect to the dilations $\left(x_{1}, x_{2}, x_{3}, x_{4}\right) \rightarrow\left(\lambda x_{1}, \lambda x_{2}, \lambda^{2} x_{3}, \lambda^{3} x_{4}\right)$. Then the derivatives $X_{1} \Phi$ and $X_{2} \Phi$ are homogeneous of degree 2 and their square roots $\left|X_{1} \Phi\right|^{1 / 2}$ and $\left|X_{2} \Phi\right|^{1 / 2}$ are homogeneous of degree 1. Analogously, $X_{1}^{2} \Phi$, $X_{2}^{2} \Phi, X_{3} \Phi$ and $\left(X_{1} X_{2}+X_{2} X_{1}\right) \Phi$, being derivatives of degree 2 , are homogeneous of degree 1 . Then the function $H=H\left(x_{1}, x_{2}, x_{3}\right)$ defined by

$$
H=\frac{\left|X_{1}^{2} \Phi\right|+\left|X_{2}^{2} \Phi\right|+\left|\left(X_{1} X_{2}+X_{2} X_{1}\right) \Phi\right|}{\left|X_{1} \Phi\right|^{1 / 2}+\left|X_{2} \Phi\right|^{1 / 2}+\left|X_{3} \Phi\right|}
$$

is homogeneous of degree 0 . We showed above that $\left|X_{1} \Phi(x)\right|^{1 / 2}+\left|X_{2} \Phi(x)\right|^{1 / 2}+$ $\left|X_{3} \Phi(x)\right|>0$ for all $N(x)>0$, and thus by 0-homogeneity

$$
\sup _{0<N(x) \leq 1 / 4} H(x)=\max _{N(x)=1 / 4} H(x)=k<+\infty .
$$

Thus Lemma 4.3 can be applied and the set $\Omega$ is admissible.

In the following examples we show that in groups of step 3 there are domains of class $C^{\infty}$ which are not John domains and for which the Sobolev-Poincaré inequality (1.1) does not hold.

We begin with a well-known lemma. Here $X=\left(X_{1}, \ldots, X_{m}\right)$ is a system of generators of a homogeneous group on $\mathbb{R}^{n}$ with homogeneous dimension $Q$. Let $d$ be the left invariant metric induced by these vector fields.

Lemma 5.3. If $\Omega \subset \mathbb{R}^{n}$ is a bounded open set such that the Sobolev-Poincaré inequality (1.1) holds, then there exist $r_{0}>0$ and $C>0$ such that

$$
|\Omega \cap B(x, r)| \geq C r^{Q}
$$

for all $x \in \bar{\Omega}$ and $0 \leq r \leq r_{0}$.

Proof. If $p=1$, (1.1) reads

$$
\left(\int_{\Omega}\left|u-u_{\Omega}\right|^{Q /(Q-1)} d x\right)^{(Q-1) / Q} \leq C \int_{\Omega}|X u| d x .
$$


Fix $\bar{x} \in \bar{\Omega}$ and $0<r<R$. The function

$$
u(x)= \begin{cases}1 & \text { if } d(x, \bar{x})<r, \\ 1-\frac{d(x, \bar{x})-r}{R-r} & \text { if } r \leq d(x, \bar{x}) \leq R, \\ 0 & \text { if } d(x, \bar{x})>R\end{cases}
$$

can be used in (5.5). Moreover, taking $R$ small enough to ensure $|\Omega \backslash B(\bar{x}, R)| \geq \frac{1}{2}|\Omega|$ we can let $u_{\Omega}=0$ to find (for a possibly different constant)

$$
\left(\int_{\Omega}|u|^{Q /(Q-1)} d x\right)^{(Q-1) / Q} \leq C \int_{\Omega}|X u| d x .
$$

Define $g(r)=|B(\bar{x}, r) \cap \Omega|$. First of all

$$
\left(\int_{\Omega}|u|^{Q /(Q-1)} d x\right)^{(Q-1) / Q} \geq g(r)^{(Q-1) / Q},
$$

and on the other hand, since $|X d(x, \bar{x})|=1$ for almost every $x$,

$$
\int_{\Omega}|X u| d x=\frac{1}{R-r}|(B(\bar{x}, R) \backslash B(\bar{x}, r)) \cap \Omega|=\frac{g(R)-g(r)}{R-r} .
$$

Thus we find

$$
g(r)^{(Q-1) / Q} \leq C \frac{g(R)-g(r)}{R-r}, \quad 0<r<R \leq r_{0} .
$$

The function $g$ is differentiable almost everywhere, being monotonic, and letting $R \rightarrow r$ in (5.6) we get $g(r)^{(Q-1) / Q} \leq C g^{\prime}(r)$ at every point of differentiability, or equivalently

$$
\frac{d}{d r} g(r)^{1 / Q} \geq \frac{1}{C}
$$

Thus

$$
g(r)^{1 / Q} \geq \int_{0}^{r} \frac{d}{d s} g(s)^{1 / Q} d s \geq \frac{1}{C} r,
$$

and the claim is proved.

Example 5.4. Consider now $\mathbb{R}^{4}$ with the homogeneous group structure introduced in Section 4 (we choose $\alpha=0$ ). The homogeneous dimension of the group is $Q=7$. Let $\Omega \subset \mathbb{R}^{4}$ be a bounded open set of class $C^{\infty}$ such that for some open neighborhood $\mathcal{U}$ of the origin $\Omega \cap \mathcal{U}=\left\{x \in \mathbb{R}^{4}: x_{4}>0\right\} \cap \mathcal{U}$. We show that the Sobolev-Poincaré inequality (1.1) fails in $\Omega$.

We localize our analysis in a neighborhood of 0 . Take a point $\bar{x}=(0,-b, 0,0) \in$ $\partial \Omega, b \neq 0$, and translate it to the origin. The translated boundary is

$$
\begin{aligned}
\left\{\bar{x}^{-1} \cdot\left(y_{1}, y_{2}, y_{3}, 0\right)\right\} & =\left\{\left(y_{1}, y_{2}+b, y_{3}+Q_{3}\left(0, b, y_{1}, y_{2}\right), Q_{4}\left(0, b, 0, y_{1}, y_{2}, y_{3}\right)\right)\right\} \\
& =\left\{\left(y_{1}, y_{2}+b, y_{3}-\frac{1}{2} b y_{1}, \frac{b}{12} y_{1}^{2}\right)\right\}=\left\{y_{4}=\frac{b}{12} y_{1}^{2}\right\} .
\end{aligned}
$$

We write $\Omega_{0}=\bar{x}^{-1} \cdot \Omega$ and assume that $b=12$ (any $b>0$ gives the same result). It will be enough to show that $\Omega_{0}$ does not support the Sobolev-Poincaré 
inequality. We can use the boxes (4.4) instead of balls to estimate for $0<r<1$,

$$
\begin{aligned}
\left|\operatorname{Box}(0, r) \cap \Omega_{0}\right| & =\int_{|y \||<r, y_{4}>y_{1}^{2}} d y \\
& =\int_{\left|y_{1}\right|<r} d y_{1} \int_{\left|y_{2}\right|<r} d y_{2} \int_{\left|y_{3}\right|<r^{2}} d y_{3} \int_{\left|y_{4}\right|<r^{3}, y_{1}^{2}<y_{4}} d y_{4} \\
& =4 r^{3} \int_{\left|y_{1}\right|<r,\left|y_{4}\right|<r^{3}, y_{1}^{2}<y_{4}} d y_{1} d y_{4} .
\end{aligned}
$$

On the integration domain we have $\left|y_{1}\right| \leq\left|y_{4}\right|^{1 / 2} \leq r^{3 / 2}$. Therefore,

$$
\left|\operatorname{Box}(0, r) \cap \Omega_{0}\right| \leq 4 r^{3} \int_{-r^{3 / 2}}^{r^{3 / 2}} d y_{1} \int_{y_{1}^{2}}^{r^{3}} d y_{4}=8 r^{3} \int_{0}^{r^{3 / 2}}\left(r^{3}-y_{1}^{2}\right) d y_{1}=\frac{16}{3} r^{15 / 2} .
$$

If the Sobolev-Poincaré inequality holds, the estimate $\left|\operatorname{Box}(0, r) \cap \Omega_{0}\right| \geq C r^{7}$ should be true, but this is not possible.

We note that $0 \in \partial \Omega$ is a characteristic point of second type which is "flat", but near 0 there are characteristic points of the same type $\bar{x}=(0,-b, 0,0), b \neq 0$, which are not "flat": by (5.7) the parametric equation for the translated boundary $\bar{x}^{-1} \cdot \partial \Omega$ is $y_{4}=\frac{b}{12} y_{1}^{2}$ and the flatness condition $\left|y_{4}\right| \leq C\left\|\left(y_{1}, y_{2}, y_{3}\right)\right\|^{3}$ does not hold. This makes $\Omega$ a bad domain.

The following example is a refined version of the previous one.

Example 5.5. In this example we disprove Conjecture 1 stated in [CG, p. 429]. We consider again the group of step 3 studied in Section 4 (with $\alpha=0$ ). We prove that in the "gauge ball"

$$
\Omega=\left\{x \in \mathbb{R}^{4}:\left(x_{1}^{2}+x_{2}^{2}\right)^{6}+x_{3}^{6}+x_{4}^{4}<1\right\}
$$

the Sobolev-Poincaré inequality does not hold. We actually show the following stronger fact.

Proposition 5.6. Assume that $\Omega \cap \mathcal{U}=\left\{x_{4}>\varphi\left(x_{1}, x_{2}, x_{3}\right)\right\} \cap \mathcal{U}$ for some neighborhood $\mathcal{U}$ of the point $(0,0,0, \varphi(0,0,0))$. Assume also that for some $\gamma>3$,

$$
\left|\varphi\left(x_{1}, x_{2}, x_{3}\right)-\varphi(0,0,0)\right| \leq C\|x\|^{\gamma}
$$

for all $x$ near the origin. Then the Sobolev-Poincaré inequality does not hold in $\Omega$.

We note that in the particular case of the homogeneous ball $\Omega=\left\{\left(x_{1}^{2}+x_{2}^{2}\right)^{6}+\right.$ $\left.x_{3}^{6}+x_{4}^{4}<1\right\}$ the function $\varphi$ is $\varphi\left(x_{1}, x_{2}, x_{3}\right)=-\left\{1-\left[\left(x_{1}^{2}+x_{2}^{2}\right)^{6}+x_{3}^{6}\right]\right\}^{1 / 4}$. Thus (5.8) is satisfied.

Proof. As in Example 5.4 we show that the estimate of Lemma 5.3 is violated, but here the choice of the center and of the radius of the balls which give the counterexample is more delicate.

Fix $\alpha \in] 1, \frac{\gamma}{3}[$ (this open interval is non-empty as soon as $\gamma>3$ ). We claim that there exist $\epsilon \in(0,1)$ and $C>0$ such that

$$
\left|\operatorname{Box}\left((0,-b, 0, \varphi(0,-b, 0)), b^{\alpha}\right) \cap \Omega\right| \leq C b^{7 \alpha+(\alpha-1) / 2} \quad \text { for all } \quad b \in(0, \epsilon) .
$$

Since $\alpha$ is strictly larger than 1 , this is not compatible as $b \rightarrow 0^{+}$with the estimate

$$
\left|\operatorname{Box}\left((0,-b, 0, \varphi(0,-b, 0)), b^{\alpha}\right) \cap \Omega\right| \gtrsim b^{7 \alpha},
$$


which by Lemma 5.3 should be satisfied if the Sobolev-Poincaré inequality would hold.

If $\bar{x}=(0,-b, 0, \varphi(0,-b, 0)), b>0$, let $\Omega_{b}=\bar{x}^{-1} \cdot \Omega$. It is easy to check that the surface $\partial \Omega_{b}:=\bar{x}^{-1} \cdot \partial \Omega$ contains the origin and can be locally parameterized by

$$
x_{4}=\varphi\left(x_{1}, x_{2}-b, x_{3}+\frac{1}{2} b x_{1}\right)-\varphi(0,-b, 0)+\frac{1}{12} b x_{1}^{2}:=\varphi_{b}\left(x_{1}, x_{2}, x_{3}\right) .
$$

If $x$ belongs to $\operatorname{Box}\left(0, b^{\alpha}\right)$, then $\left|x_{1}\right|,\left|x_{2}\right| \leq b^{\alpha}$ and $\left|x_{3}\right|<b^{2 \alpha}$. Thus we have the estimate

$$
\left|\varphi\left(x_{1}, x_{2}-b, x_{3}+\frac{1}{2} b x_{1}\right)\right| \lesssim\left|x_{1}\right|^{\gamma}+\left|x_{2}-b\right|^{\gamma}+\left|x_{3}+\frac{1}{2} b x_{1}\right|^{\gamma / 2} \lesssim b^{\gamma},
$$

because $\alpha>1$. Moreover, $|\varphi(0,-b, 0)| \leq b^{\gamma}$.

This implies that if $x \in \operatorname{Box}\left(0, b^{\alpha}\right) \cap \Omega_{b}$, then $x_{4}>\varphi_{b}\left(x_{1}, x_{2}, x_{3}\right)$ and

$$
\begin{aligned}
\frac{1}{12} b x_{1}^{2} & \leq\left|x_{4}\right|+|\varphi(\ldots)-\varphi(\ldots)| \\
& \lesssim b^{3 \alpha}+b^{\gamma} \simeq b^{3 \alpha},
\end{aligned}
$$

because $3 \alpha<\gamma$. As a consequence $\left|x_{1}\right| \lesssim b^{(3 \alpha-1) / 2}$ and

$$
\operatorname{Box}\left(0, b^{\alpha}\right) \cap \Omega_{b} \subset\left\{\left|x_{1}\right|<C_{0} b^{(3 \alpha-1) / 2},\left|x_{2}\right|<b^{\alpha},\left|x_{3}\right|<b^{2 \alpha},\left|x_{4}\right|<b^{3 \alpha}\right\}
$$

for some $C_{0}$ which stays bounded as $b$ approaches 0 . This proves (5.9)).

\section{ACKNOWLEDGMENTS}

We would like to thank Nicola Garofalo who has drawn our attention to the problems studied in Section 3, Olli Martio who has discussed with us some general properties of John and uniform domains and Nicoletta Ripamonti for her help with the computer. Finally, we wish to thank Bruno Franchi and Luca Capogna for pointing out some references to us.

\section{REFERENCES}

[Be] O. V. Besov, Integral representations of functions in a domain with the flexible horn condition, and imbedding theorems (Russian), Dokl. Akad. Nauk. SSSR 273 (1983), 1294-1297. MR0731291 (85i:46036)

[Bo] B. Bojarski, Remarks on Sobolev imbedding inequalities, in Proc. of the Conference on Complex Analysis (Joensu 1987), 52-68, Lecture Notes in Math. 1351, Springer, 1988. MR0982072 (90b:46068)

[BKL] S. Buckley, P. Koskela, G. Lu, Boman equals John, XVIth Rolf Nevanlinna Colloquium (Joensuu, 1995), 91-99, de Gruyter, Berlin, 1996. MR.1427074 (98m:43013)

[CG] L. Capogna, N. Garofalo, Boundary behavior of nonnegative solutions of subelliptic equations in NTA domains for Carnot-Carathéodory metrics, J. Fourier Anal. Appl. 4 (1998), 4-5, 403-432. MR1658616 (2000k:35056)

[CGN1] L. Capogna, N. Garofalo, D. M. Nhieu, Examples of uniform and NTA domains in Carnot groups, Proceedings on Analysis and Geometry (Russian) (Novosibirsk Akademgorodok, 1999), 103-121, Izdat. Ross. Akad. Nauk Sib. Otd. Inst. Mat., Novosibirsk, 2000. MR 1847513 (2002k:30037)

[CGN2] L. Capogna, N. Garofalo, D. M. Nhieu, Properties of harmonic measures in the Dirichlet problem for nilpotent Lie Groups of Heisenberg type, Amer. J. Math. 124 (2002), 273306. MR 1890994 (2002m:31013)

[CT] L. Capogna, P. Tang, Uniform domains and quasiconformal mappings on the Heisenberg group, Manuscripta Math. 86 (1995), no. 3, 267-281. MR.1323792|(96f:30019) 
[DGN] D. Danielli, N. Garofalo, D. M. Nhieu, Non-doubling Ahlfors measures, perimeter measures and the characterization of the trace spaces of Sobolev functions in CarnotCarathéodory spaces, preprint.

[FF1] F. Ferrari, B. Franchi, A local doubling formula for harmonic measure associated with subelliptic operators, Comm. Partial Differential Equations 28 (2003), 1-60. MR1974448 (2004g:35050)

[FF2] F. Ferrari, B. Franchi, Geometry of the boundary and doubling property of the harmonic measure for Grushin type operators, Rend. Sem. Mat. Univ. Politec. Torino 58 (2002), 281-299. MR 1984194 (2004g:35049)

[FS] G. B. Folland, E. M. Stein, Hardy spaces on homogeneous groups, Princeton University Press, 1982. MR0657581 (84h:43027)

[FLW] B. Franchi, G. Lu, R. L. Wheeden, Representation formulas and weighted Poincaré inequalities for Hörmander vector fields, Ann. Inst. Fourier 45 (1995), 577-604. MR.1343563 (96i:46037)

[GN1] N. Garofalo, D. M. Nhieu, Isoperimetric and Sobolev inequalities for Carnot-Carathéodory spaces and the existence of minimal surfaces, Comm. Pure Appl. Math. 49 (1996), 1081-1144. MR,1404326 (97i:58032)

[GN2] N. Garofalo, D. M. Nhieu, Lipschitz continuity, global smooth approximations and extension theorems for Sobolev functions in Carnot-Carathéodory spaces, J. Anal. Math. 74 (1998), 67-97. MR1631642 (2000i:46025)

[G] A. V. Greshnov, On uniform and NTA-domains on Carnot groups, (Russian) Sibirsk. Mat. Zh. 42 (2001), no. 5, 1018-1035, ii. Mr.1861631 (2002h:53048)

[HK] P. Hajłasz, P. Koskela, Sobolev met Poincaré, Mem. Amer. Math. Soc. 688 (2000). MR 1683160 (2000j:46063)

$[\mathrm{HH}]$ W. Hansen, H. Hueber, The Dirichlet problem for sub-Laplacians on nilpotent Lie groups - geometric criteria for regularity, Math. Ann. 276 (1987), no. 4, 537-547. MR 0879533 (88g:31017)

[J] D. Jerison, The Poincaré inequality for vector fields satisfying Hörmander's condition, Duke Math. J. 53 (1986), 503-523. MR0850547 (87i:35027)

[JK] D. Jerison, C. E. Kenig, Boundary behavior of harmonic functions in non-tangentially accessible domains, Adv. Math. 46 (1982), 80-147. MR0676988 (84d:31005b)

[Joh] F. John, Rotation and strain, Comm. Pure Appl. Math. 4 (1961), 391-414. MR0138225 $(25: 1672)$

[Jon] P. W. Jones, Quasiconformal mappings and extendability of functions in Sobolev spaces, Acta Math. 147 (1981), 71-88. MR0631089 (83i:30014)

[MS] O. Martio, J. Sarvas, Injectivity theorems in plane and space, Ann. Acad. Sci. Fenn., Ser. A I Math. 4 (1978/1979) 383-401. MR0565886 (81i:30039)

[VSC] N. Th. Varopoulos, L. Saloff Coste and T. Coulhon, Analysis and Geometry on Groups, Cambridge Univ. Press, 1992. MR1218884 (95f:43008)

[V] J. Väisälä, Uniform domains, Tohoku Math. J. 40 (1988), 101-118. MR0927080 (89d:30027)

[VG] S. K. Vodop'yanov, A. V. Greshnov, On extension of functions of bounded mean oscillation from domains in a space of homogeneous type with intrinsic metric, Siberian Math. J. 36 (1995), no. 5, 873-901. MR,1373594 (97j:42010)

[W] R. Wittmann, A nontangential limit theorem, Osaka J. Math. 24 (1987), no. 1, 61-76. MR0881746 (88g:31021)

Dipartimento di Matematica Pura ed Applicata, Università di Padova, via Belzoni 7 , 35131 PADOVA, ITALY

E-mail address: monti@math.unipd.it

Dipartimento di Matematica, Università di Bologna, Piazza di Porta San Donato, 5, 40127 BOLOGNA, ITALY

E-mail address: morbidel@dm.unibo.it 